ARTICLE

\title{
Differential Rac1 signalling by guanine nucleotide exchange factors implicates FLII in regulating Rac1-driven cell migration
}

Hadir Marei ${ }^{1}$, Alejandro Carpy ${ }^{2}$, Anna Woroniuk $^{1, \star}$, Claire Vennin $^{3, \star}$, Gavin White $^{1, \star}$, Paul Timpson ${ }^{3}$, Boris Macek $^{2} \&$ Angeliki Malliri ${ }^{1}$

The small GTPase Rac1 has been implicated in the formation and dissemination of tumours. Upon activation by guanine nucleotide exchange factors (GEFs), Rac1 associates with a variety of proteins in the cell thereby regulating various functions, including cell migration. However, activation of Rac1 can lead to opposing migratory phenotypes raising the possibility of exacerbating tumour progression when targeting Rac1 in a clinical setting. This calls for the identification of factors that influence Rac1-driven cell motility. Here we show that Tiam1 and P-Rex1, two Rac GEFs, promote Rac1 anti- and pro-migratory signalling cascades, respectively, through regulating the Rac1 interactome. In particular, we demonstrate that P-Rex1 stimulates migration through enhancing the interaction between Rac1 and the actin-remodelling protein flightless-1 homologue, to modulate cell contraction in a RhoA-ROCK-independent manner.

\footnotetext{
${ }^{1}$ Cell Signalling Group, Cancer Research UK Manchester Institute, The University of Manchester, Manchester M204BX, UK. ${ }^{2}$ Proteome Center Tuebingen, Interfaculty Institute for Cell Biology, University of Tuebingen, Tuebingen 72026, Germany. ${ }^{3}$ Invasion and Metastasis Group, Garvan Institute of Medical Research, The Kinghorn Cancer Centre, Faculty of Medicine, St Vincent's Clinical School, University of New South Wales, Darlinghurst, New South Wales 2010, Australia. * These authors contributed equally to this work. Correspondence and requests for materials should be addressed to A.M. (email: Angeliki.Malliri@cruk.manchester.ac.uk).
} 
C ell migration and invasion play an important role in various physiological functions, such as embryonic development, immune cell responses and wound healing. In addition, aberrant regulation of cell motility is linked to tumour progression with increased cell migration and invasion marking a key step in metastasis, the major cause of death in cancer patients $^{1,2}$. Studies focused on deciphering the underlying mechanisms involved in cell migration and invasion have identified five key steps that govern the mesenchymal-mode of single-cell motility: (1) front-rear polarization; (2) membrane protrusion at the leading edge; (3) cell-extra-cellular matrix (ECM) adhesion; (4) actomyosin contractility; and (5) detachment of the cell rear $^{3,4}$. Given the required interplay between the actin cytoskeleton, cell-ECM adhesions and myosin motor contractile forces, various signalling proteins are implicated in regulating cell migration. Rac1, a member of the Rho family of small GTPases, is of particular importance. Similarly to other small GTPases, Rac1 serves as a molecular switch cycling between an inactive GDP-bound form and an active GTP-bound form. Upon activation by guanine nucleotide exchange factors (GEFs), Rac1 undergoes a conformational change allowing it to bind to and activate downstream effectors that directly and indirectly influence cell migration and invasion in normal and cancer cells $s^{3,5,6}$. However, activation of Rac1 can lead to opposing migratory phenotypes. Through its ability to promote front-rear polarization ${ }^{7,8}$, lamellipodia formation ${ }^{9-11}$, as well as assembly of focal complexes at the leading edge ${ }^{12,13}$, Rac1 promotes cell migration and invasion. However, Rac1 is also essential for maintaining cell-cell contacts in epithelial cells via promoting cadherin-mediated cell-cell junctions ${ }^{14-16}$. As a result, Racl is implicated in impeding cell migration and invasion. The dual role of Rac1 in cell migration and invasion is further demonstrated by its ability to regulate the expression and release of matrix metalloproteinases ${ }^{17,18}$, which aid in ECM degradation, as well as the matrix metalloproteinase inhibitors, tissue inhibitor of metalloproteinases ${ }^{19}$. These contrasting roles of Racl in migration and invasion make its therapeutic potential uncertain and call for the identification of factors that regulate Rac1 downstream specificity.

Racl differential downstream effects are often attributed to differences in cell type and upstream signalling from the $\mathrm{ECM}^{20,21}$; however, the mechanism by which these inputs control selectivity downstream of Racl is poorly understood. Interestingly, GEFs have been shown to influence responses downstream of Rho1 in yeast cells ${ }^{22,23}$. Moreover, a limited number of studies propose a scaffolding role of GEFs, whereby they bind either directly to GTPase effectors ${ }^{24-26}$ or indirectly through other scaffolding proteins ${ }^{27,28}$. Thus GEFs present an interesting class of proteins that could potentially play a role not only in Racl activation but also in dictating Racl downstream effects that govern its anti-migratory versus pro-migratory cellular phenotypes.

To rigorously evaluate the hypothesis that GEFs determine output downstream of Racl by regulating its interaction with effectors, we were particularly interested in comparing Rac1 GEFs known to induce opposing Racl-driven cellular effects. We therefore focused on Tiam1 and P-Rex1, two Rac GEFs that have been associated with contrasting migratory phenotypes ${ }^{14,29-33}$. Interestingly, we show that activation of Rac1 by either GEF, under the same cellular conditions, results in distinct morphological phenotypes and differential actin cytoskeletal rearrangements that dictate Racl anti- versus pro-migratory roles. In addition, we performed a quantitative mass spectrometry screen uncovering distinct sets of interactors with differential Racl binding, dependent on the upstream GEF. Focusing on P-Rexl-enriched Rac1 binding partners, we identify protein flightless-1 homolog (FLII), a gelsolin protein superfamily member, as a novel Racl effector that is required for mediating P-Rex1-Rac1-driven cell migration through modulating cell contraction in a RhoA-ROCK-independent manner. Thus, our data demonstrate the importance of GEFs in dictating Rac1 functional specificity through modulating effector binding and uncover a previously unreported signalling cascade that regulates Rac1-driven cell migration.

\section{Results}

Tiam1 and P-Rexl induce differential Racl downstream effects. To achieve selective activation of Tiam1-Rac1 and P-Rex1-Rac1 signalling cascades irrespective of upstream signalling, we utilized a doxycycline (dox)-inducible system to overexpress wild-type (WT) Tiam1 or P-Rex1 in NIH3T3 cells. In addition, to exclude phenotypic changes due to GEF overexpression alone rather than GEF-mediated Rac1 activation, previously described Tiam1 and P-Rex1 GEF-dead mutants $\left(G^{*} F^{\star}\right)^{34,35}$ were also introduced into cells and analysed in parallel with WT proteins (Supplementary Fig. 1a). Active Rac1 pulldown experiments demonstrated the ability of Tiam1 WT and P-Rex1 WT but not their GEF$^{*}$ mutants to activate Racl (Supplementary Fig. 1b,c). In addition, unlike Rac1, levels of active Cdc42 and RhoA, two small GTPases that are also implicated in cell migration, were not affected upon expression of the different GEF constructs, confirming the selectivity of Tiam1 and P-Rex1 in activating Rac1 (Fig. 1a,b).

Changes in cell morphology and actin cytoskeleton rearrangements are known to accompany cell migration ${ }^{4}$, therefore using the dox-inducible system we examined the effect of GEFs in regulating these processes. In NIH3T3 cells, expression of Tiam1 WT was associated with increased cell aggregation and membrane ruffling inducing an epithelial-like morphology in the otherwise mesenchymal NIH3T3 cells (Fig. 1c,d). These morphological changes were also accompanied with increased actin localization at cell-cell contacts, consistent with the observed cell aggregation (Supplementary Fig. 1d). More importantly, expression of Tiam1 WT resulted in a significant reduction in cell migration (Fig. 1e,f). In contrast, expression of P-Rex1 WT was predominantly associated with an elongated morphology and the formation of thin membrane protrusions (Fig. 1c,d) rich in polymerized actin (Supplementary Fig. 1d). In addition, Oris migration assays demonstrated that unlike Tiam1 WT, P-Rex1 activation of Rac1 resulted in significantly increased cell migration (Fig. 1e,f). Importantly, these observations were limited to dox treated cells expressing the WT proteins but not the $\mathrm{GEF}^{\star}$ mutants (Fig. 1 and Supplementary Fig. 1), indicating that the observed phenotypes following expression of Tiam 1 and P-Rex1 are Rac1-driven.

Interestingly, expression of Tiam1 WT and P-Rex1 WT, but not their $\mathrm{GEF}^{*}$ mutants, in the epithelial squamous cell carcinoma cell line, A431, and the epithelial immortalized Madin-Darby canine kidney cell line, MDCKII, resulted in phenotypic effects similar to those observed in NIH3T3 cells (Fig. 2 and Supplementary Fig. 2). Expression of Tiam1 WT induced an even more compact epithelioid morphology associated with membrane ruffling and more defined cell-cell contacts that were distinguishable from control cells (Fig. 2a,b and Supplementary Fig. 2a,b). Consistently, increased actin localization at cell junctions was observed in A431 cells expressing Tiam1 WT (Fig. 2c). On the other hand, P-Rex1 WT expression resulted in a prominent morphological shift to elongated cells (Fig. 2a,b and Supplementary Fig. 2a,b), with disrupted actin staining at cell-cell contacts (Fig. 2c). GEF expression was also accompanied by differential cell migration in MDCKII cells, with Tiam1 WT significantly suppressing migration and P-Rexl WT promoting migration (Supplementary 
a

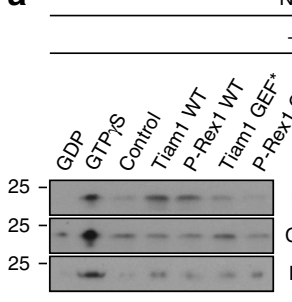

Active GTPase pulldown
NIH3T3

+ Dox

$$
\text { 岕 }
$$

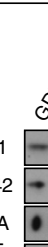

RhoA $1--0-0$

$245=$
180
$245=$
180
$63-$

$---1-1$

Input

\section{b}

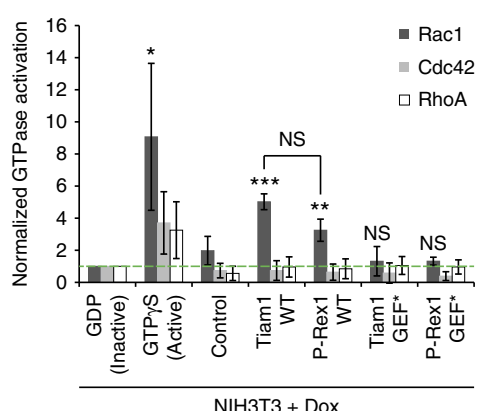

NIH3T3 + Dox

C
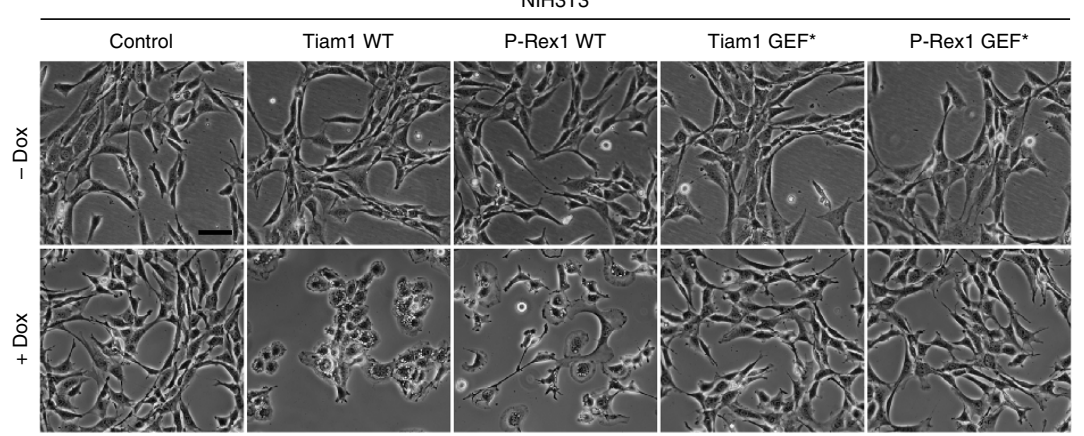

d - Elongated $\square$ Epithelial-like - Mesenchymal

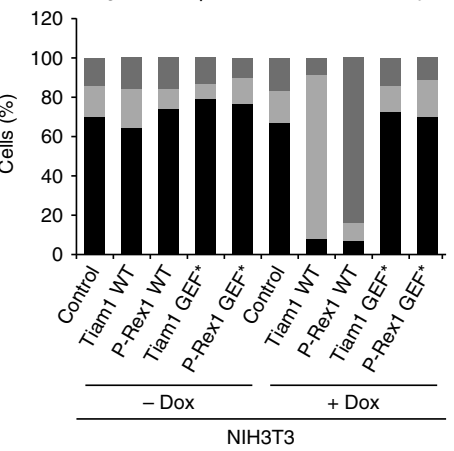

$\mathbf{f}$

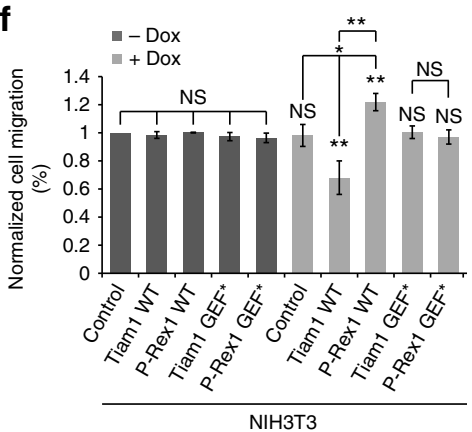

e
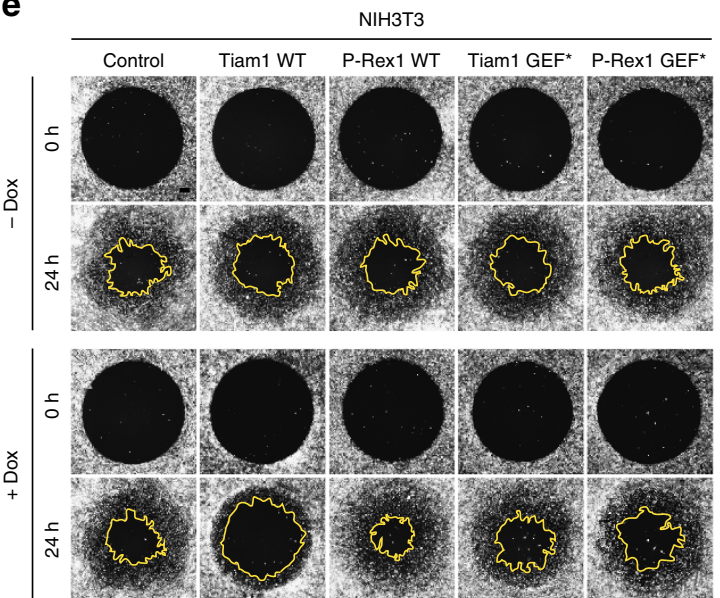

Figure 1 | Activation of Rac1 by Tiam1 or P-Rex1 induces differential effects in NIH3T3 cells. (a) Lysates from NIH3T3 cells following $1 \mu$ g ml ${ }^{-1}$ doxycycline (dox) treatment for $24 \mathrm{~h}$ to induce expression of indicated GEF constructs were divided equally and subjected to PAK-CRIB pulldown or Rhotekin pulldown. Levels of active and total Rac1, Cdc42 and RhoA were detected by western blot analysis. GDP and GTP $\gamma$ S loaded NIH3T3 lysates were used as negative and positive pulldown controls, respectively. $\alpha$-Tubulin was used as a loading control. (b) Quantification of levels of active Rac1, Cdc42 and RhoA in NIH3T3 cells described in a relative to total levels of the respective proteins normalized to GDP-loaded NIH3T3 lysates \pm s.e.m. from three independent experiments. The dashed green line indicates basal levels of active GTPases in the GDP control. (c) Representative phase-contrast images of $\mathrm{NIH} 3 \mathrm{~T} 3$ cells treated with ethanol ( - dox) or $1 \mu \mathrm{g} \mathrm{ml} \mathrm{m}^{-1}$ dox ( + dox) for $24 \mathrm{~h}$ to induce expression of indicated GEF constructs. Scale bar, $100 \mu \mathrm{m}$.

(d) Quantification of GEF-induced cellular phenotypes depicted in c. Graph represents per cent cells with the indicated morphology from a total of 150 cells per condition from three independent experiments. (e) Representative fluorescence images following Oris migration assay of NIH3T3 cells treated with ethanol ( - dox) or $1 \mu \mathrm{g} \mathrm{ml}^{-1}$ dox ( + dox) for $24 \mathrm{~h}$ to induce expression of indicated GEF constructs. Scale bar, $200 \mu \mathrm{m}$. (f) Quantification of cell migration of NIH3T3 cells described in e normalized to - dox treated control cells. Graph represents the average per cent migration \pm s.e.m from three independent experiments. In b,f, student's $t$-test was used to assess significance as indicated on graphs. $P$ values indicated above each bar are relative to GDP (b) or - dox treated control cells (f). NS, non-significant; ${ }^{\star}=P \leq 0.05 ;{ }^{\star \star}=P \leq 0.01 ;{ }^{\star \star \star}=P \leq 0.001$. 
a A431

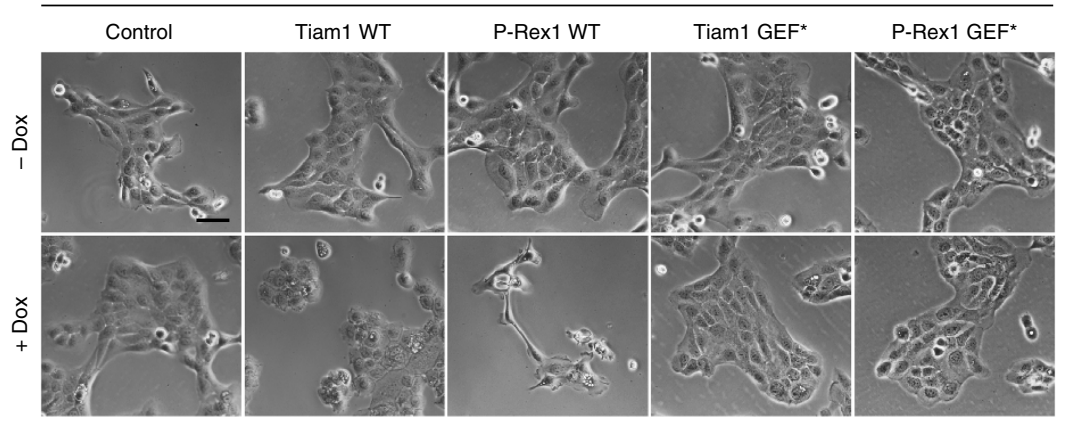

b

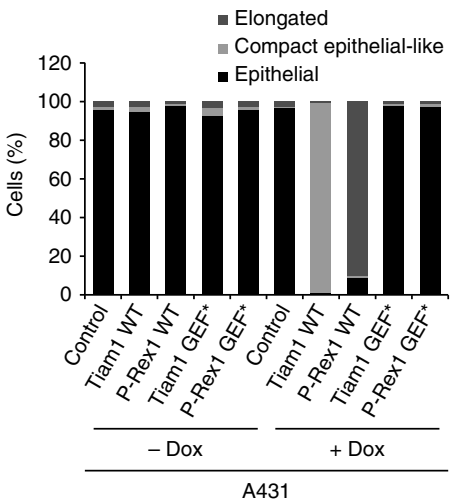

d

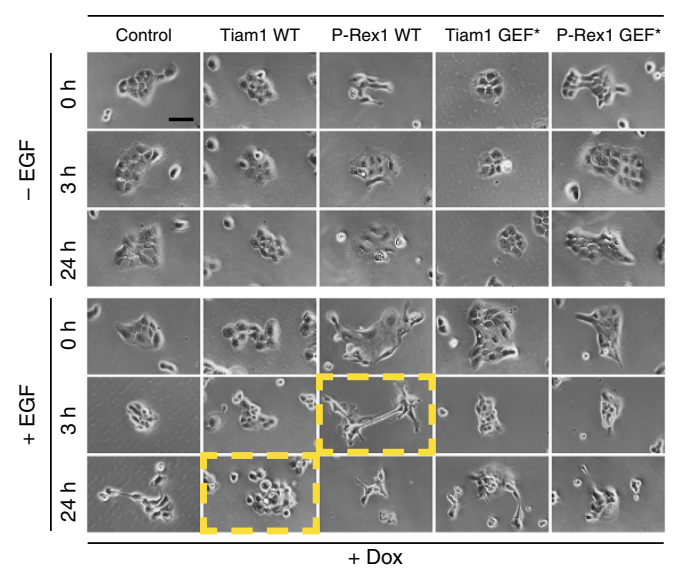

C

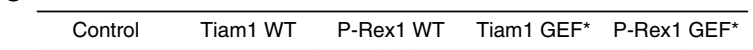

+ Dox

e

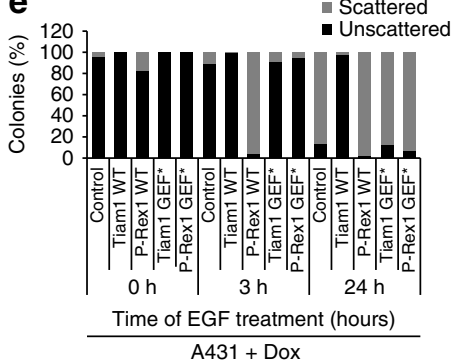

f

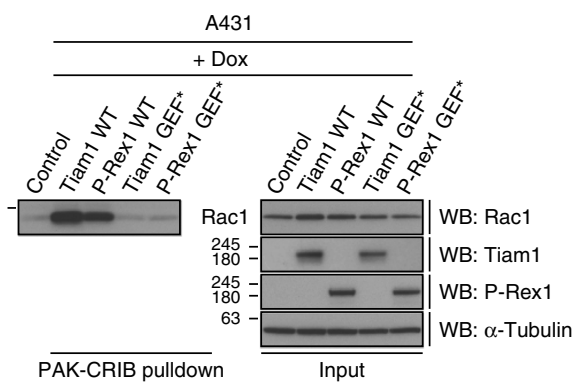

Figure 2 | Activation of Rac1 by Tiam1 or P-Rex1 induces differential effects in A431 cells. (a) Representative phase-contrast images of A431 cells treated with ethanol ( - dox) or $1 \mu \mathrm{g} \mathrm{ml}{ }^{-1}$ doxycycline ( + dox) for $24 \mathrm{~h}$ to induce expression of indicated GEF constructs. Scale bar, $100 \mu \mathrm{m}$. (b) Quantification of GEF-induced cellular phenotypes depicted in a. Graph represents per cent cells with the indicated morphology from a total of 150 cells per condition from three independent experiments. (c) Representative immunofluorescence images of + dox treated A431 cells fixed in $4 \%$ formaldehyde. Phalloidin staining was used to visualize the actin cytoskeleton and antibodies against HA-tagged Tiam1 WT and GEF* or Myc-tagged P-Rex1 WT and GEF* to detect the expression of the respective GEFs upon dox induction. DAPI was used to stain the nuclei. Scale bar, $100 \mu \mathrm{m}$. (d) Representative phase-contrast images of + dox treated A431 cells either left untreated ( $-E G F$ ) or treated with $100 \mathrm{ng} \mathrm{ml}^{-1}$ epidermal growth factor ( + EGF) for the indicated times following serum starvation for 18-24 h. Yellow boxes are used to highlight the differential effects of Tiam1 WT and P-Rex1 WT on cell scattering at indicated time points. Scale bar, $100 \mu \mathrm{m}$. (e) Quantification of cell scattering of + dox treated A431 cells described in d. Graph represents per cent of scattered or unscattered colonies from a total of 50 colonies per condition from three independent experiments. (f) Lysates from A431 cells following + dox treatment for $24 \mathrm{~h}$ to induce expression of indicated GEF constructs were subjected to PAK-CRIB pulldown. Levels of active and total Rac1 were detected by western blot analysis. $\alpha$-Tubulin was used as a loading control. Representative western blot from three independent experiments. 
Fig. 2c,d). Moreover, Tiam1 WT expression impeded epidermal growth factor (EGF)-induced cell scattering in A431 cells and hepatocyte growth factor (HGF)-induced cell scattering in MDCKII cells, while P-Rex1 WT expression resulted in enhanced scattering detected as early as $3 \mathrm{~h}$ post growth factor treatment (Fig. 2d,e and Supplementary Fig. 2e,f). As expected and similarly to NIH3T3 cells, elevated levels of active Rac1 were only detected upon expression of the WT but not the GEF* mutants (Fig. $2 \mathrm{f}$ and Supplementary Fig. 2g) further confirming the dependency of Tiam 1 and P-Rex1 on Racl to induce differential phenotypes. Taken together these data demonstrate the ability of Tiam 1 and P-Rex1, irrespective of upstream signalling, to induce cell morphological changes and actin cytoskeletal rearrangements that govern Rac1-driven anti- and pro-migratory phenotypes, respectively, in both normal and cancer cell lines.

Tiam1 and P-Rex1 differentially regulate the Racl interactome. Having demonstrated that Tiam1 and P-Rex1 elicit distinct Rac1driven phenotypes, we were interested in deciphering the mechanism by which these contrasting phenotypes are manifested. We hypothesized that the observed GEF-induced differential cell migratory phenotypes might be mediated by the ability of Tiam 1 and P-Rex1 to modulate Rac1-effector binding. To test this hypothesis, we engineered a double dox-inducible system for expression of StrepII-FLAG-tagged Rac1 (SF-Rac1) alone or together with either Tiam1 or P-Rex1 (WT or GEF* mutants) in NIH3T3 cells. We then coupled the efficiency of SF-tandem affinity purification (TAP) $)^{36-38}$ with the quantitative power of stable isotope labelling by amino acids in cell culture (SILAC) ${ }^{39,40}$ to examine the Racl interactome following induction of either Tiam1 or P-Rex1. By designing a five-way SILAC screen we were able to directly compare the relative abundance of Rac1associated proteins upon expression of Tiam1 WT/GEF* or P-Rex1 WT/GEF* to cells expressing SF-Rac1 only (control). Given that the same control cells were used to identify changes in the Racl interactome between WT GEF expression (Set1) and $\mathrm{GEF}^{*}$ mutant expression (Set2), we were also able to indirectly assess the effect of WT versus GEF* GEF expression on Rac1effector binding. In addition, to increase the robustness of the SILAC quantification, we performed reverse SILAC in which the SILAC labelling media were switched between Tiam1 WT/GEF* and P-Rex1 WT/GEF* cells (Supplementary Fig. 3a).

Combined, 350 proteins were identified as putative Rac1 interacting proteins from a total of two SILAC and two reverse SILAC screens. We first assessed the ability of the SF-TAP/SILAC approach to identify bona fide Racl binding partners via conducting an extensive literature review combined with searching available protein databases, such as the human protein reference database (ref. 41), NetPath (ref. 42) and the human protein-protein interaction predictions database ${ }^{43,44}$, to analyse the full list of identified proteins. As expected the SILAC screen yielded several known Racl binding partners, predicted Racl interactors, as well as proteins implicated in Rac1 signalling (Supplementary Table 1), thus suggesting that a large number of the remaining proteins are genuine novel Racl interactors.

From the identified proteins, 231 were associated with quantifiable SILAC ratios in $\geq 2$ SILAC screens. Interestingly, using a commonly accepted cutoff of \pm 1.3 fold-change ${ }^{45}$, we identified a subset of Racl interactors that showed changes in Racl binding only upon expression of either GEF (Supplementary Fig. 3b), thus highlighting a role of Tiam 1 and P-Rex1 in differentially modulating the Rac1 interactome.

FLII is a novel P-Rexl-enriched Racl binding partner. To determine whether GEF-mediated regulation of the Racl interactome is important for inducing differential effects downstream of Rac1, we further classified the identified Racl binding partners according to their cellular functions using the Ingenuity integrated pathway analysis (IPA) software. Given the observed GEFinduced differential effects on cell migration, we focused on a shortlist of differentially regulated proteins falling within functional categories that relate to cell motility, including cell movement, cell morphology, cell signalling, cell-to-cell signalling and interaction, as well as cell assembly and organization (Fig. 3a). Among the identified proteins, FLII exhibited a significant GEF-dependent Rac1 binding fold-change between Tiam1 WT and P-Rex1 WT expressing cells. Relative to NIH3T3 cells expressing SF-Rac1 alone, SILAC ratios indicated that FLII was associated with approximately a five-fold $(P=1.46 \times 10$ $-6)$ and a 10 -fold $(P=7.13 \times 10-9)$ increase in SF-Rac1 binding in a forward and reverse SILAC experiment, respectively, that was specific to P-Rex1 WT. In addition, FLII was not co-purified with SF-Rac1 upon expression of either Tiam1 GEF* or P-Rex1 GEF*, suggesting that it is functionally important for P-Rex1-Rac1-driven cellular effects. In contrast, the known Rac1 interactor RhoGDI1 showed limited SILAC ratio fluctuations across the different samples that were comparable to ratios observed for Rac1 itself (Supplementary Fig. 3c), indicating that the relative abundance of RhoGDI1 protein associated with Rac1 does not change upon expression of the different GEF constructs. Taken together, these data demonstrate that SILAC ratios associated with proteins identified from the screens are not due to fluctuations in Racl purification, and more importantly that expression of P-Rex1 WT induces specific changes in FLII SILAC ratios that are not observed for other proteins, such as RhoGDI1.

FLII is a member of the actin-remodelling gelsolin protein superfamily and has been implicated in cell migration. It has been shown to translocate from the nucleus and perinuclear region in motile NIH3T3 cells to actin-rich regions at the cell periphery, including membrane ruffles and the leading edge ${ }^{46}$. Moreover, FLII is implicated in regulating focal adhesion turnover through inhibiting the phosphorylation of paxillin in a Rac1-dependent manner ${ }^{47}$, providing a link between FLII and Racl signalling. Using western blot analysis, we confirmed that exogenous SF-Rac1 co-precipitates endogenous FLII following a streptavidin (Strep) pulldown of SF-Rac1 from HEK293T cells (Fig. 3b). In addition, endogenous FLII also co-precipitated endogenous Racl confirming that FLII is a novel Racl binding partner (Fig. 3c). Furthermore, GST-pulldown experiments with purified GST or GST-Rac1 loaded with either GDP or GTP $\gamma S$ and incubated with endogenous FLII from HEK293T lysates revealed that FLII binds preferentially to active Rac1 (Fig. 3d,e).

To further validate this interaction, we utilized the Duolink in situ proximity ligation assay (PLA) to visualize the interaction between endogenous Racl and endogenous FLII upon expression of the different GEFs in NIH3T3 cells. Interestingly, an increased Duolink signal was only detected upon expression of P-Rex1 WT (Fig. 3f,g). We also investigated the effect of P-Rexl WT overexpression in MCF7 cells, which express higher levels of endogenous P-Rex1 compared with NIH3T3 cells, thus presenting a cell line that is configured to respond physiologically to P-Rex1 signalling. Similarly to NIH3T3 cells, overexpression of P-Rex1 WT in MCF7 cells stimulated the endogenous Rac1-FLII interaction as indicated by the increased Duolink signal detected only in P-Rex1 WT expressing cells (Supplementary Fig. 3d,e). Pulldown of SFRac1 from MCF7 cells following SF-TAP also revealed an enhanced interaction with endogenous FLII upon expression of P-Rex1 WT but not the other GEF constructs. In contrast, there was no change in the levels of co-precipitated RhoGDI1 upon expression of the different GEF constructs (Supplementary Fig. 3f,g). Together, the Duolink in situ PLA assays and the SF-TAP further validate the 
a

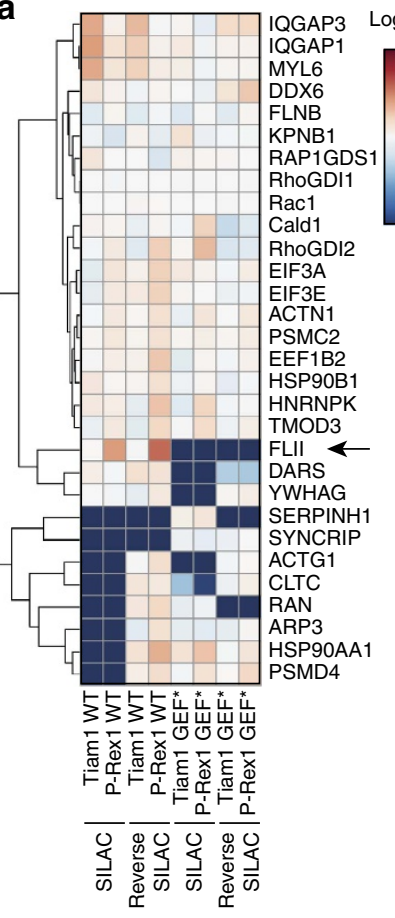

b

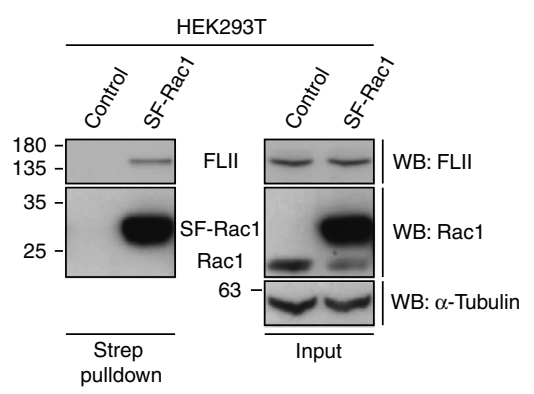

d

GST

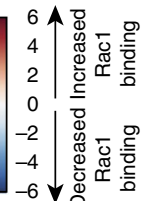

HEK293T
C

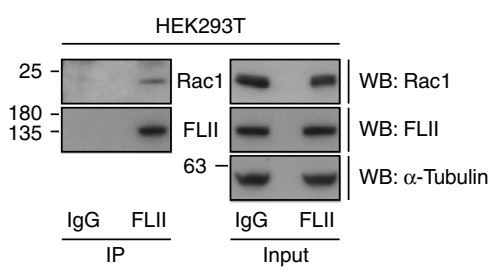

e

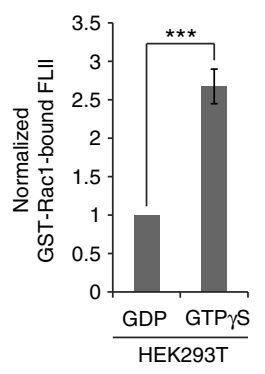

f

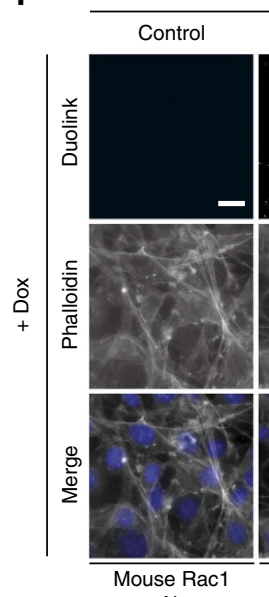

$\mathrm{Ab}$

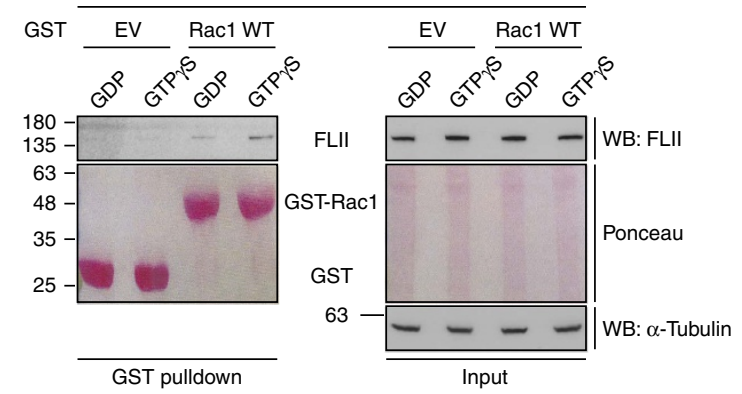

NIH3T3

\section{g}

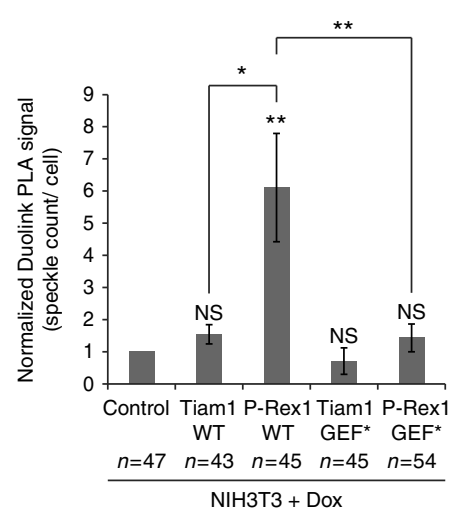

Figure 3 | FLII is a novel P-Rex1-enriched Rac1 interactor that binds preferentially to active Rac1. (a) Heat map of indicated SILAC identified Rac1 interactors classified under one of the following Ingenuity integrated pathway analysis (IPA) functional groups: cell movement, cell morphology, cell signalling, cell-to-cell signalling and interaction, as well as cell assembly and organization. The heat map represents the Log2 of SILAC protein ratios relative to control cells expressing SF-Rac1 alone from a representative SILAC and reverse SILAC screen. (b) Streptavidin (Strep) pulldown from HEK293T cells expressing SF-Rac1. Co-precipitated endogenous FLII was detected by western blot analysis. (c) Endogenous FLII immunoprecipitation (IP) from HEK293T cells. Co-precipitated endogenous Rac1 was detected by western blot analysis. In b,c, $\alpha$-Tubulin was used as a loading control. Representative western blots from three independent experiments. (d) GST pulldown using purified GST (EV) or GST-tagged Rac1 WT (Rac1 WT) loaded with GDP or GTP $\gamma$ S and incubated with HEK293T lysates. Co-precipitated endogenous FLII was detected by western blot analysis. $\alpha$-Tubulin and ponceau staining were used as loading controls. (e) Quantification of endogenous GST-Rac1-bound FLII in HEK293T cells described in d normalized to GDP-loaded GST-Rac1 WT \pm s.e.m. from three independent experiments. Student's $t$-test was used to assess significance as indicated on graph. ${ }^{\star \star \star}=P \leq 0.001$. (f) Representative immunofluorescence images of NIH3T3 cells fixed in $4 \%$ formaldehyde and subjected to the Duolink in situ PLA assay following treatment with $1 \mu \mathrm{mg}$ ml ${ }^{-1}$ doxycycline ( + dox $)$ for $24 \mathrm{~h}$ to induce expression of indicated GEF constructs. Phalloidin and DAPI were used to visualize the actin cytoskeleton and nuclei, respectively. Scale bar, $20 \mu \mathrm{m}$. (g) Quantification of average Duolink PLA signal from indicated number of NIH3T3 cells described in $\mathbf{f} \pm$ s.e.m. Student's $t$-test was performed to determine statistical significance and $P$ values are shown on graph. $P$ values indicated above each bar are relative to control cells. NS, non-significant; ${ }^{\star}=P \leq 0.05 ;{ }^{\star \star}=P \leq 0.01$.

SILAC screen and confirm that the Racl-FLII interaction is enhanced upon P-Rex1 WT expression.

FLII is a novel P-Rexl binding partner. Given the proposed role of GEFs in controlling GTPase signalling through acting as molecular scaffolds, we were interested in determining whether, in addition to Rac1, FLII binds to P-Rex1 and not Tiam1. Immunoprecipitation of FLAG-tagged FLII from HEK293T cells co-expressing Myc-Tiam1 WT or Myc-P-Rex1 WT revealed that FLII binds preferentially to P-Rex1 (Fig. 4a). Similarly, Myc-PRex1 WT but not Myc-Tiam1 WT co-precipitated FLAG-tagged FLII (Supplementary Fig. 4a). The P-Rex1-FLII interaction 
a

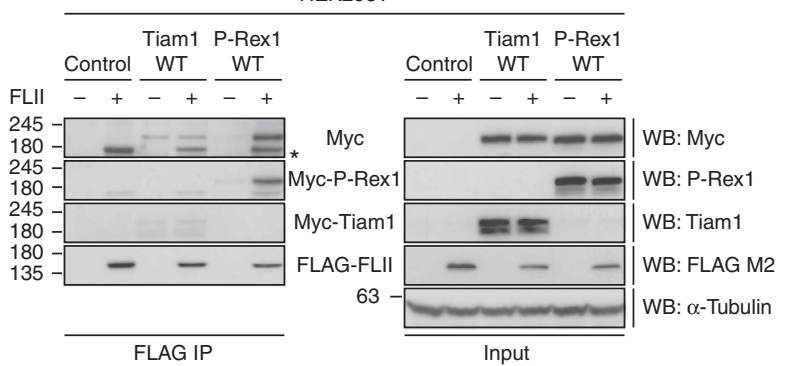

b

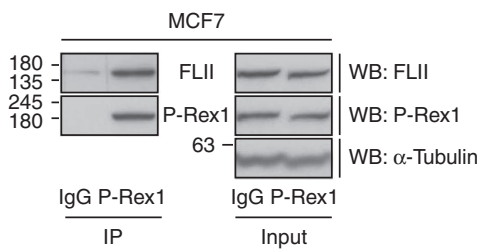

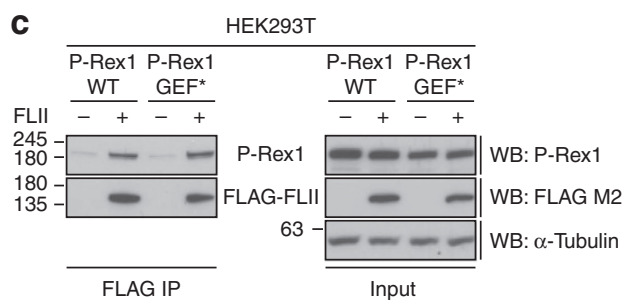

Figure 4 | FLII is a novel P-Rex1 interactor that does not bind to Tiam1. (a) FLAG immunoprecipitation (IP) from HEK293T cells expressing Myc-tagged Tiam1 WT or Myc-tagged P-Rex1 WT alone or together with FLAG-tagged FLII. Co-precipitated Myc-tagged GEFs were detected by western blot analysis. * indicates background band. (b) Endogenous P-Rex1 IP from MCF7 cells. Co-precipitated endogenous FLII was detected by western blot analysis. (c) FLAG IP from HEK293T cells expressing Myc-tagged P-Rex1 WT or GEF* alone or together with FLAG-tagged FLII. Co-precipitated Myc-tagged GEFs were detected by western blot analysis. In $\mathbf{a}-\mathbf{c}, \boldsymbol{\alpha}$-Tubulin was used as a loading control. Representative western blots from three independent experiments.

was also confirmed on an endogenous level using MCF7 cells following immunoprecipitation of endogenous P-Rex1 (Fig. 4b) or endogenous FLII (Supplementary Fig. 4b). P-Rex1 has been shown to drive cell migration and invasion in a number of melanoma cell lines, including CHL1 (ref. 32). Thus, to address whether this interaction occurs in a cellular setting in which P-Rex1 promotes migration and invasion we utilized the Duolink in situ PLA assay to visualize P-Rex1-FLII binding in CHL1 cells. Interestingly, there was a significant increase in the Duolink signal in CHL1 cells when both the P-Rex1 and FLII antibodies were applied compared with cells subjected to the FLII antibody only, indicating a strong endogenous interaction in these cells (Supplementary Fig. 4c,d). Moreover, we found that both P-Rex1 WT and P-Rex1 GEF* bind to FLII in equal levels, demonstrating that the P-Rex1-FLII interaction is independent of the P-Rex1 GEF activity (Fig. 4c) potentially occurring upstream of Rac1 activation. Although GEF activity is not a prerequisite for P-Rex1-FLII interaction, GTP-loading of Rac1 enhances the Rac1 interaction with FLII. This hints at a dual role of P-Rex1 as both a Rac1 GEF and a molecular scaffold.

FLII binds to Racl and P-Rexl through different domains. As a member of the gelsolin protein superfamily, FLII possesses the characteristic gelsolin-like (GEL) domain. However, unlike other superfamily members, FLII also contains an N-terminal leucinerich repeats (LRR) domain (Fig. 5a) with the two domains governing distinct functions. The GEL domain is mainly responsible for FLII-mediated actin remodelling ${ }^{48}$. On the other hand, LRR domains serve as protein-protein interaction motifs via forming a doughnut- or horseshoe-like conformation that serves as a hydrophobic pocket for protein binding ${ }^{49-51}$. To gain functional insight into the Rac1-FLII and P-Rex1-FLII interactions, previously described FLAG-tagged full-length FLII (FLII FL), a GEL only mutant (FLII GEL), and an LRR only mutant (FLII LRR) (ref. 52) (Fig. 5a) were used to determine the FLII domain responsible for mediating its interaction with Rac1 and P-Rex1. Intriguingly, FLAG co-immunoprecipitation of the different FLII domain mutants revealed that P-Rex1 binds to the GEL domain (Fig. 5b), whereas Rac1 binds preferentially to the LRR domain of FLII (Supplementary Fig. 5a). Using purified proteins we showed that P-Rex1 binds directly to the GEL domain of FLII (Supplementary Fig. 5b). Rac1 binding to the LRR domain of FLII was also further confirmed by GST pulldown of purified GTP $\gamma$ S loaded GST-Racl incubated with lysates from HEK293T cells expressing the different FLAG-tagged FLII domain mutants (Fig. 5c). Together, the above data imply that P-Rexl, Racl and FLII might form a ternary complex in cells that is functionally important for eliciting P-Rex1-Rac1-driven cellular phenotypes.

FLII is required for P-Rex1-Rac1-driven cell migration. Despite a negative role for FLII in cell migration having previously been described ${ }^{53-56}$, P-Rex1 promotes migration through Rac1 activation concomitant with increased Rac1-FLII binding, raising the possibility that FLII might positively influence migration. FLII depletion by siRNA in both - dox and + dox treated control NIH3T3 cells was associated with a modest yet significant decrease in the ability of cells to migrate when compared with mock and non-targeting (NT) treated cells (Supplementary Fig. 6a). Similar results were observed in -dox treated NIH3T3 cells transduced with the different GEF dox-inducible systems (Supplementary Fig. 6b-d and Fig. 6a-c). Interestingly, compared with mock and NT treated cells, there was no additive effect of FLII knockdown on the ability of Tiam1 WT to reduce cell migration in NIH3T3 cells (Supplementary Fig. 6b). FLII depletion in Tiam1 GEF* and P-Rex1 GEF* expressing cells, on the other hand, resulted in a modest yet significant reduction in cell migration (Supplementary Fig. 6c,d) similar to that observed in control NIH3T3 cells (Supplementary Fig. 6a). Importantly, FLII depletion was sufficient to abolish the ability of P-Rex1 WT to stimulate cell migration (Fig. 6a-c).

To further understand the role of FLII in P-Rex1-Rac1-driven cell migration we performed single-cell tracking following scratch assays in NIH3T3 cells treated with dox to induce expression of P-Rex1 WT. Consistent with the ORIS migration assays described above, FLII depletion using two different siRNAs was associated 
a

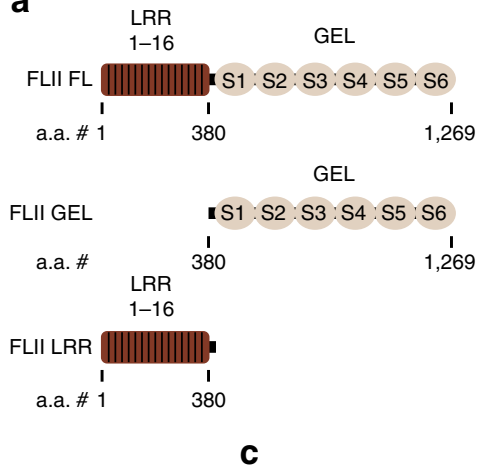

b

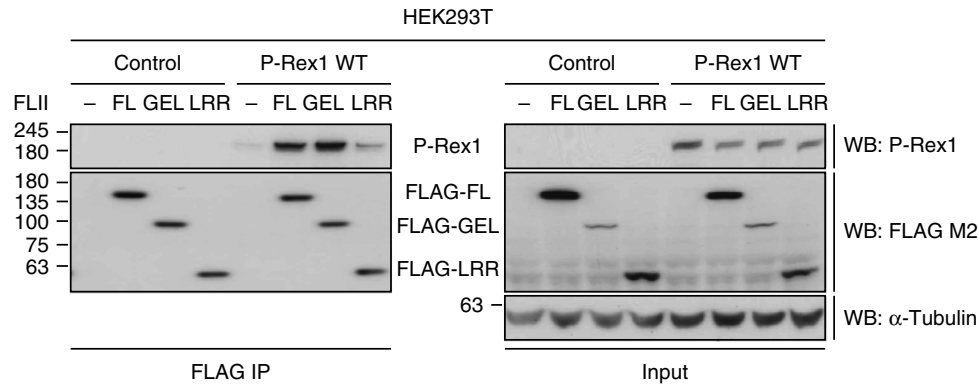

HEK293T

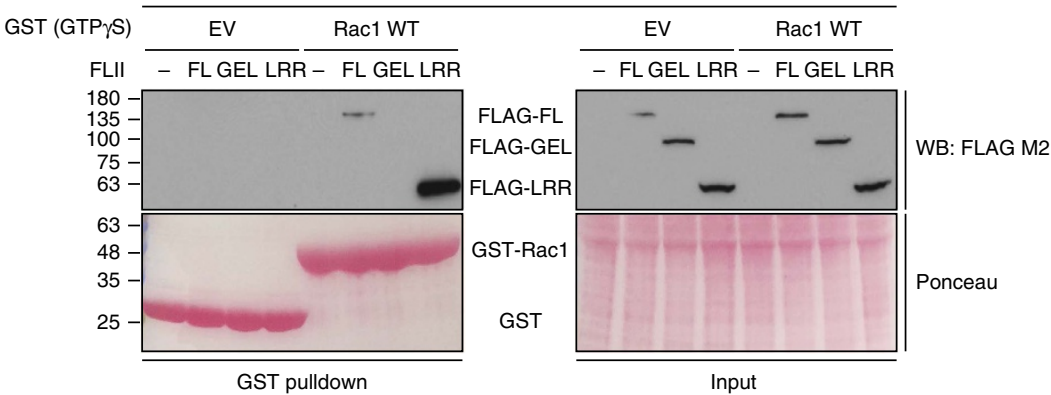

Figure 5 | P-Rex1 and Rac1 bind preferentially to the GEL and LRR domain of FLII, respectively. (a) Schematic representation of FLII domain structure showing 16 leucine-rich repeats (LRR) comprising the N-terminal LRR domain and six gelsolin-like repeats (S1-S6) representing the C-terminal GEL domain together with the amino acid number (a.a. \#) range for each domain. FLII FL, full-length FLII; FLII GEL, gelsolin domain only; FLII LRR, LRR domain only. (b) FLAG immunoprecipitation (IP) from HEK293T cells expressing the different FLAG-tagged FLII domain mutants outlined in a alone or together with P-Rex1 WT. Co-precipitated exogenous P-Rex1 was detected by western blot analysis. $\alpha$-Tubulin was used as a loading control. Representative western blot from three independent experiments. (c) GST pulldown using purified GST (EV) or GST-tagged Rac1 WT (Rac1 WT) loaded with GTP $\gamma$ S and incubated with HEK293T lysates expressing the different FLAG-tagged FLII domain mutants outlined in a. Co-precipitated FLAG-tagged FLII domain mutants were detected by western blot analysis. Ponceau staining was used as a loading control. Representative western blot from three independent experiments.

with a significant decrease in cell migration as measured by various parameters, including accumulated distance, cell displacement and speed (Fig. 6d-f). Interestingly, FLII knockdown had no effect on the straightness of the track of cell movement (Fig. 6g) indicating that while FLII is important for mediating P-Rex1-driven cell migration it does not influence directionality. Active Rac1 pulldown experiments following FLII depletion in NIH3T3 cells expressing P-Rex1 WT also demonstrated that the diminished migratory capacity of FLII knockdown cells is not due to reduced levels of either total or active Rac1 in these cells (Supplementary Fig. 6e,f). Importantly, levels of active RhoA were not affected upon expression of P-Rex1 WT in confluent monolayers of NIH3T3 cells that were scratched to induce cell migration (Supplementary Fig. 6g,h). Similarly, there was no significant increase in the levels of active RhoG in NIH3T3 cells expressing P-Rex1 WT upon stimulation of migration following scratching (Supplementary Fig. 6i) suggesting that P-Rexl acts directly on Racl and not through RhoA or RhoG to stimulate cell migration in a FLII-dependent manner.

Knockdown of FLII was also associated with reduced cell migration in CHL1 cells (Fig. $6 \mathrm{~h}-\mathrm{j}$ ), indicating that FLII is important for optimal cell migration in a cellular setting in which endogenous P-Rex 1 is known to drive cell migration and invasion $^{32}$. Interestingly, in migrating CHL1 cells both P-Rex 1 and FLII co-localize at the leading edge together with actin (Supplementary Fig. 6j). All together, these data highlight the interplay between P-Rex1, Racl and FLII that is required for optimal P-Rex1-Rac1-driven cell migration.

FLII is required for P-Rex1-induced cell contractility. FLII has been shown to regulate cell contraction ${ }^{47}$. Activation of myosin II through myosin light chain (MLC) phosphorylation induces actomyosin contractility that is crucial for mediating cell migration ${ }^{4}$. The level of phosphorylated myosin II at the cell centre and rear is mediated through RhoA-ROCK signalling ${ }^{57}$; however, two recent studies have implicated Racl in regulating myosin II phosphorylation and localization at the leading edge $^{58,59}$. This combined with our findings demonstrating that GEF expression does not induce RhoA activation in NIH3T3 cells hinted at a potential role of P-Rex1 in promoting contractility in a FLII-dependent manner. To explore this possibility, primary $\left(1^{\circ}\right)$ human fibroblasts expressing pRetroX-Tight-Pur empty vector (EV), Tiam1 WT or P-Rex1 WT were mixed with acid extracted rat-tail collagen $I$ and the ability of either GEF to mediate contraction was assessed using a fibroblast-collagen matrix contraction assay as previously described ${ }^{60}$. Interestingly, expression of P-Rex1 WT, but not Tiam1 WT enhanced fibroblast-collagen matrix contraction significantly compared with the - dox treated and EV controls (Fig. 7a,b). This was further demonstrated by examining the levels of phosphorylated MLC (pMLC) upon expression of either GEF. We found that expression of P-Rex1 WT but not Tiam1 WT resulted in a significant increase in levels of pMLC compared with the EV control (Supplementary Fig. 7a,b). Consistently, immunohistochemistry of fibroblast-collagen matrices following expression of the different constructs showed a significant increase in the number of pMLC positive cells, only upon expression of P-Rex1 WT (Supplementary Fig. 7c). Importantly, depletion of endogenous P-Rex1 in both CHL1 and MCF7 cells was associated with a significant reduction in pMLC levels (Fig. 7c,d and Supplementary Fig. 7d,e). Consistent with the increased contractile nature of P-Rex1 WT expressing fibroblasts, 
a

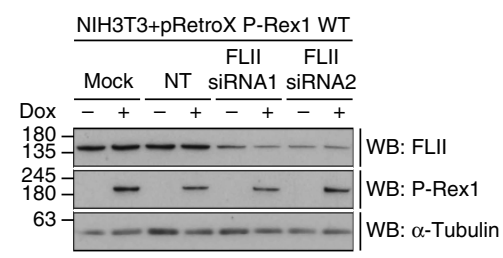

C

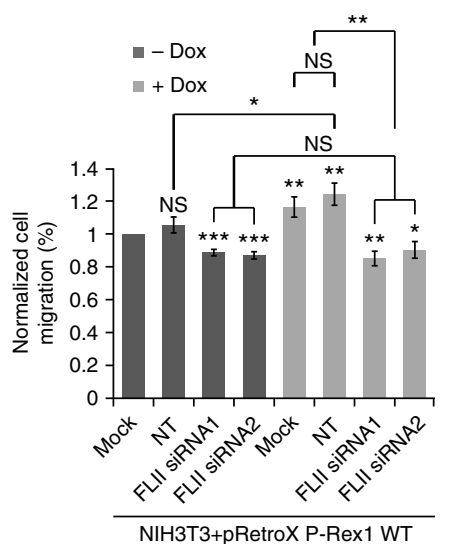

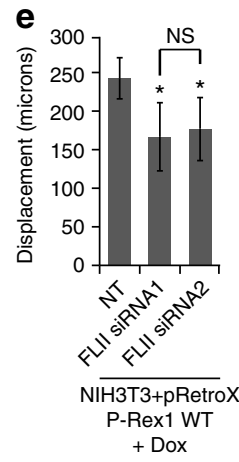

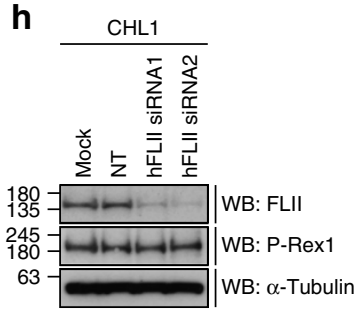

b

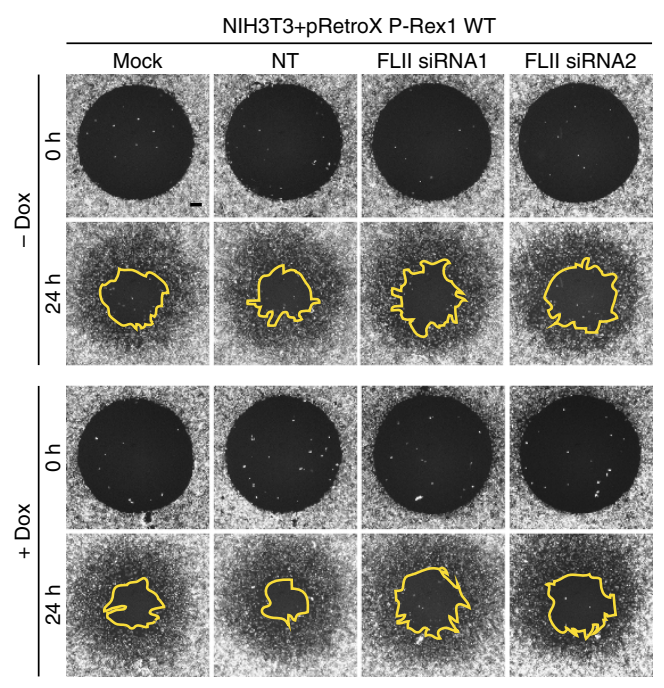

i

f
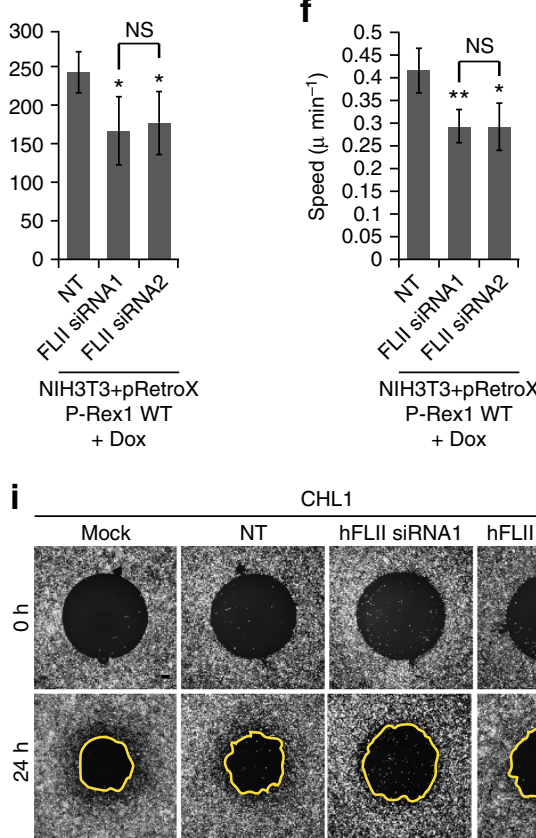
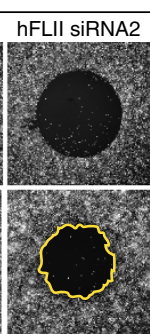

g

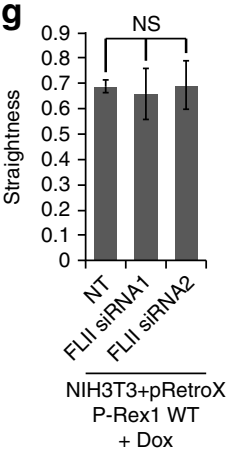

j

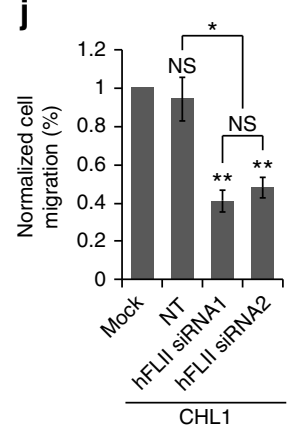

Figure 6 | FLII is required for P-Rex1-Rac1-driven cell migration. (a) Western blot of NIH3T3 cells (NIH3T3 + pRetroX P-Rex1 WT) treated with mock, NT or two different siRNAs against FLII (FLII siRNA1 and FLII siRNA2) in the presence of ethanol ( - dox) or $1 \mu g$ ml ${ }^{-1}$ doxycycline $(+$ dox) to induce expression of P-Rex1 WT. Levels of endogenous FLII and P-Rex1 expression were detected by western blot analysis. (b) Representative fluorescence images following Oris migration assay of cells described in a. Scale bar, $200 \mu \mathrm{m}$. (c) Quantification of cell migration of NIH3T3 cells described in a normalized to - dox treated mock cells. (d-g) Single-cell tracking of NIH3T3 cells expressing P-Rex1 WT following dox induction and treatment with indicated oligos. Graphs show the effect of FLII knockdown on (d) accumulated distance, (e) displacement, (f) speed and (g) straightness. (h) Western blot of CHL1 cells transfected with NT oligo or two different siRNAs against human FLII (hFLII siRNA1 and hFLII siRNA2). Levels of endogenous FLII were detected by western blot analysis. In $\mathbf{a}, \mathbf{h} \alpha$-Tubulin was used as a loading control. Representative western blots from three independent experiments. (i) Representative fluorescence images following Oris migration assay of CHL1 cells treated with indicated oligos. Scale bar, $200 \mu \mathrm{m}$. (j) Quantification of cell migration of $\mathrm{CHL} 1$ cells described in $\mathbf{h}$ normalized to mock treated cells. For $\mathbf{c}, \mathbf{j}$ graphs represent the average per cent migration \pm s.e.m. from three independent experiments. For $\mathbf{c}-\mathbf{g}, \mathbf{j}$, Student's $t$-test was used to assess significance as indicated on graphs. $P$ values indicated above each bar are relative to - dox treated mock cells $(\mathbf{c})$, or NT control $(\mathbf{d}-\mathbf{g})$ or mock treated cells $(\mathbf{j})$. NS $=$ non-significant; ${ }^{*}=P \leq 0.05 ;{ }^{\star \star}=P \leq 0.01 ;{ }^{\star \star \star}=P \leq 0.001$.

only fibroblast-collagen matrices prepared using P-Rex1 WT expressing $1^{\circ}$ human fibroblasts exhibited increased collagen content and crosslinking as indicated by picrosirius staining and second harmonic generation (SHG) imaging, respectively (Fig. 7e,f and Supplementary Fig. 7f,g). Additionally, we utilized gray-level co-occurrence matrix (GLCM) analysis ${ }^{61}$ on 
a

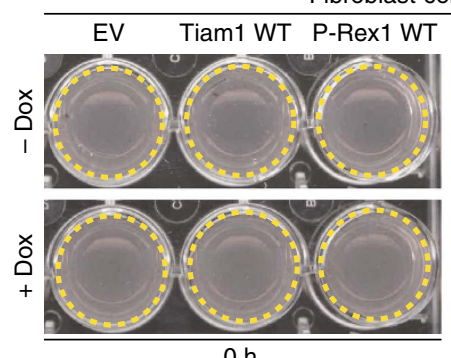

Fibroblast-collagen matrix

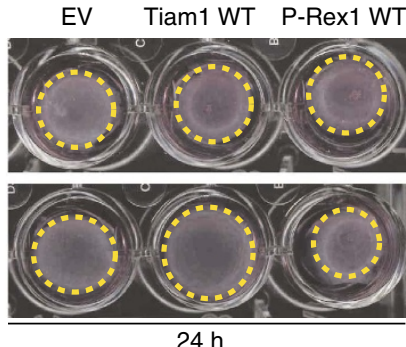

b

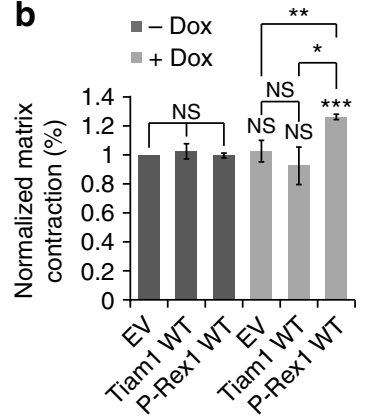

C

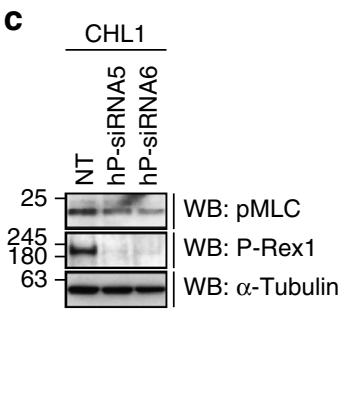

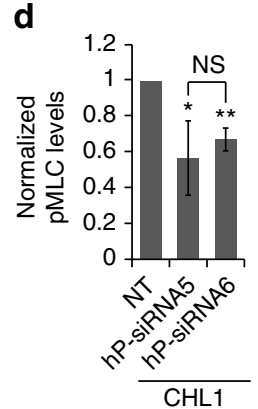

e

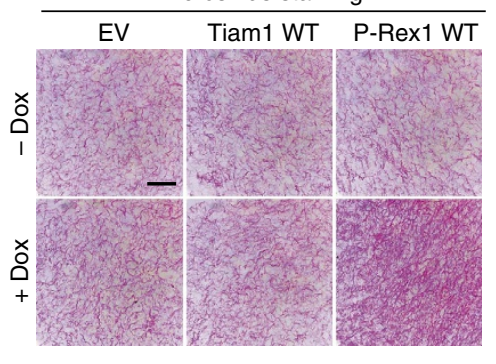

i

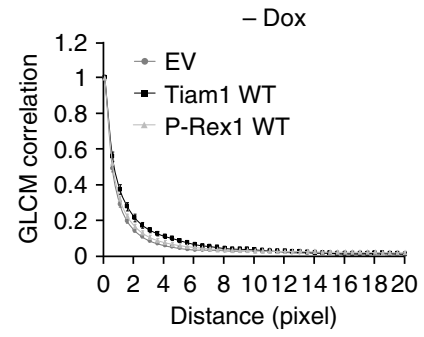

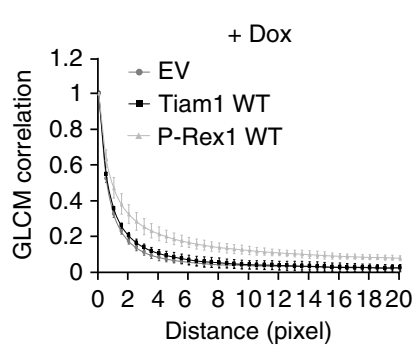

f

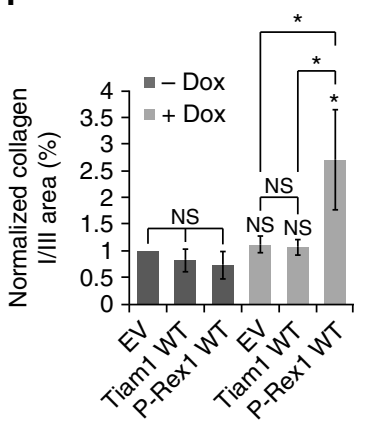

j

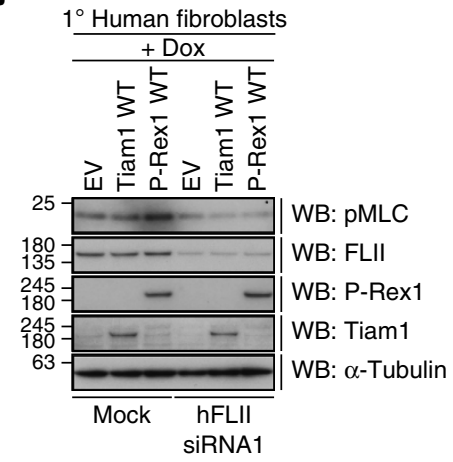

g

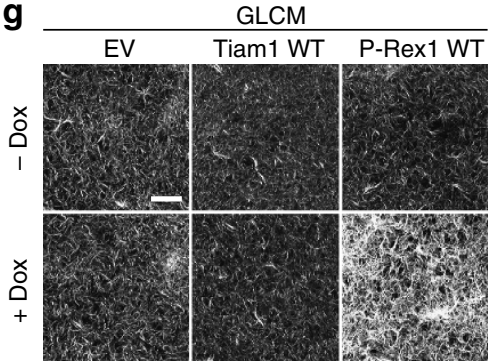

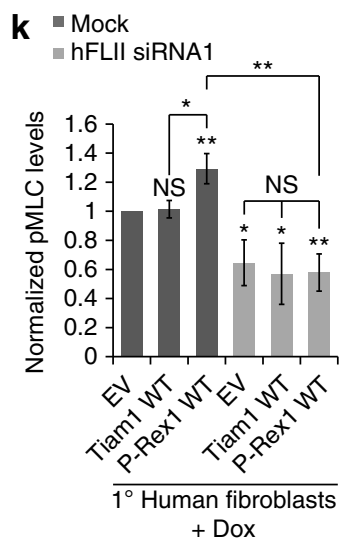

Figure 7 | FLII is required for P-Rex1-driven cell contraction. (a) Fibroblast-collagen matrices were treated with ethanol ( - dox $)$ or $1 \mu g$ ml ${ }^{-1}$ doxycycline $(+$ dox $)$ to induce expression of indicated constructs and scans were taken at 0 and $24 \mathrm{~h}$ post treatment. (b) Quantification of cell contraction depicted in a normalized to - dox treated EV control. Graph represents the average per cent matrix contraction \pm s.e.m. from three independent experiments. (c) CHL1 cells were transfected with NT, or two different siRNAs against human P-Rex1 (hP-siRNA5, hP-siRNA6). Levels of endogenous pMLC and P-Rex1 were detected by western blot analysis. (d) Quantification of pMLC levels in CHL1 cells described in c normalized to $\alpha$-Tubulin and pMLC levels detected in the NT control. Graph represents average pMLC levels \pm s.e.m. from three independent experiments. (e) Collagen picrosirius staining of matrices described in a. Scale bar, $50 \mu \mathrm{m}$. (f) Quantification of picrosirius staining depicted in e normalized to - dox treated EV control. Graph represents the area covered by collagen I and III \pm s.e.m. from three independent experiments. (g) Representative GLCM images of matrices described in a. Scale bar, $100 \mu \mathrm{m}$. (h,i) GLCM correlation decay curves of matrices described in a showing - dox (h) and + dox (i) curves. (j) $1^{\circ}$ human fibroblasts were either mock transfected or treated with siRNA against human FLII (hFLII siRNA1) in the presence of dox to induce expression of indicated constructs. Levels of endogenous pMLC and FLII were detected by western blot analysis. For $\mathbf{c}, \mathbf{j}, \alpha$-Tubulin was used as a loading control. (k) Quantification of pMLC levels in $1^{\circ}$ human fibroblasts described in $\mathbf{j}$ normalized to $\alpha$-Tubulin and pMLC levels detected in the EV control. Graph represents average pMLC levels \pm s.e.m. from three independent experiments. For $\mathbf{b}, \mathbf{d}, \mathbf{f}, \mathbf{k}$, Student's t-test was used to assess significance as indicated on graphs. $P$ values indicated above each bar are relative to - dox treated EV control $(\mathbf{b}, \mathbf{f})$, NT control $(\mathbf{d})$ or mock treated EV control $(\mathbf{k})$. NS, non-significant; ${ }^{\star}=P \leq 0.05 ;{ }^{\star \star}=P \leq 0.01 ;{ }^{\star \star \star}=P \leq 0.001$.

fibroblast-collagen matrices following 9 days of GEF expression to examine changes in collagen remodelling. As suggested by the picrosirius staining and SHG imaging, expression of P-Rex1 WT but not Tiam1 WT was associated with increased collagen crosslinking and remodelling (Fig. $7 \mathrm{~g}-\mathrm{i}$ ). We next examined the dependency of P-Rex1-mediated cell contraction on FLII. Interestingly, FLII depletion by siRNA in $1^{\circ}$ human fibroblasts was associated with an overall reduction in pMLC levels regardless of which construct was expressed. More importantly, FLII knockdown in P-Rex1 WT expressing cells inhibited P-Rex1
WT-induced MLC phosphorylation (Fig. 7j,k). This data highlights a FLII-dependent role of P-Rexl in regulating cell contraction, thus uncovering a novel signalling cascade by which P-Rex1 can mediate Rac1-driven cell migration through FLII.

Cell contraction regulates $\mathbf{P}$-Rex1-driven cell migration. To further explore the role of cell contractility in P-Rex1-Rac1-FLIIdriven cell migration we performed ORIS migration assays using NIH3T3 cells expressing P-Rex1 WT upon dox induction that 
were either left untreated (control) or treated with an inactive enantiomer of blebbistatin [( + )-blebbistatin], blebbistatin $[( \pm)$ blebbistatin], or a ROCK inhibitor (Y27632). Interestingly, blebbistatin treatment was associated with a significant reduction in P-Rex1-driven cell migration, while the ROCK inhibitor had no effect on cell migration (Fig. 8a,b). Similarly, treatment of CHL1 cells with blebbistatin but not the inactive enantiomer or the ROCK inhibitor impeded migration (Fig. 8c,d), indicating that RhoA-ROCK-independent cell contraction is required for optimal P-Rex1-driven cell migration in an endogenous setting. Taken together, these results indicate that P-Rex1 promotes cell contractility and consequently migration through Racl and FLII in a RhoA-ROCK-independent manner.

\section{Discussion}

When considering the contribution of Racl signalling to cancer dissemination, a challenge facing the field is accounting for its contrasting effects on migration and invasion. This conundrum can be resolved by defining contexts eliciting a given behaviour and identifying factors that dictate Racl downstream signalling cascades that govern its anti- and pro-migratory roles. Work presented here provides direct evidence implicating GEFs in modulating Racl downstream signalling. Through the use of a

controlled cell system in which GEF expression is the only variable, we demonstrate that expression of Tiam 1 and P-Rex1 induces distinct phenotypes in three different cell lines. In addition, via assessing the role of $\mathrm{GEF}^{*}$ mutants of both proteins, we show that these effects are Racl-driven. Moreover, the quantitative proteomic screen we undertook has allowed us to begin to dissect the mechanisms by which GEFs regulate the Rac1 interactome to mediate Racl anti- and pro-migratory effects. This is demonstrated by the role we now show for FLII in regulating P-Rex1-driven cell contraction, which accounts, at least in part, for P-Rex1-Rac1-mediated cell migration (Fig. 8e).

Through our work, we confirm that Tiam1 activation of Rac1 stimulates cell-cell adhesion and reduces cell migration. This was particularly evident in the mesenchymal NIH3T3 cells in which we show that expression of Tiam1 induces an epithelial-like morphology accompanied by reduced cell migration, as previously described by Sander et al. ${ }^{29}$ A similar phenotypic switch is also seen in metastatic melanoma cells, in which Tiam1 is shown to impede cell migration through regulating the actin cytoskeleton to promote cell-cell contacts and induce an epithelial-like morphology ${ }^{30}$. In addition, we demonstrate that Tiam1 not only antagonizes HGF-induced cell scattering of MDCKII cells, as reported by Hordijk et al. ${ }^{14}$, but also impedes EGF-induced scattering of A431 cells. Together these results

C
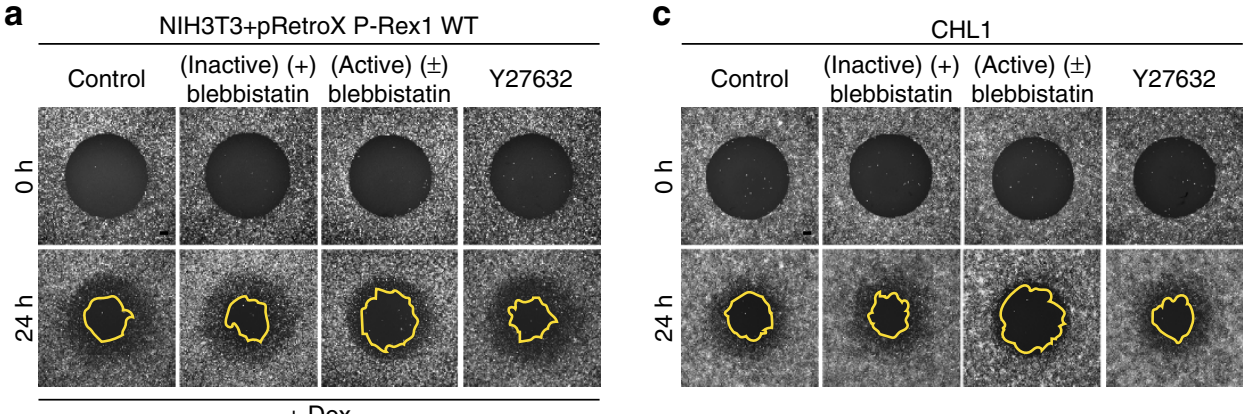

b

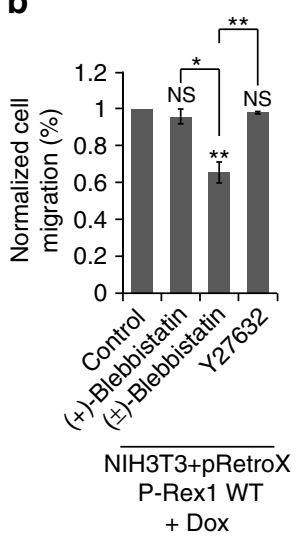

d

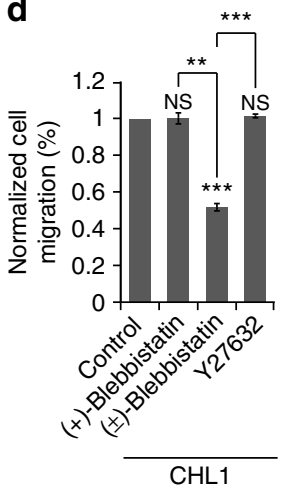

e

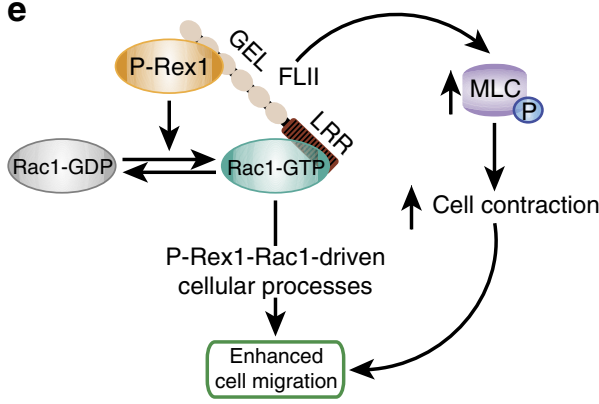

Figure 8 | RhoA-ROCK-independent cell contraction is required for P-Rex1-Rac1-FLII-driven cell migration. (a) Representative fluorescence images following Oris migration assay of NIH3T3 cells either left untreated (control), or treated with $100 \mu \mathrm{M}$ inactive enantiomer of blebbistatin $[(+)$ blebbistatin], $100 \mu \mathrm{M}$ blebbistatin [( \pm ) blebbistatin] or $10 \mu \mathrm{M}$ ROCK inhibitor (Y27632) in the presence of $1 \mu \mathrm{g} \mathrm{ml}{ }^{-1}$ doxycycline ( + dox) to induce expression of P-Rex1 WT. Scale bar, $200 \mu \mathrm{m}$. (b) Quantification of cell migration of NIH3T3 cells described in a normalized to control cells. (c) Representative fluorescence images following Oris migration assay of CHL1 cells either left untreated (control) or treated with indicated chemicals. Scale bar, $200 \mu \mathrm{m}$. (d) Quantification of cell migration of CHL1 described in c normalized to control cells. For b,d, graphs represent the average per cent migration \pm s.e.m. from three independent experiments. Student's $t$-test was used to assess significance as indicated on graphs. $P$ values indicated above each bar are relative to control cells. NS, non-significant; ${ }^{\star}=P \leq 0.05 ;{ }^{\star \star}=P \leq 0.01 ;{ }^{\star \star \star}=P \leq 0.001$. (e) Schematic representation of the P-Rex1-Rac1-FLII signalling cascade. Activation of Rac1 by P-Rex1 results in Rac1 binding to a set of downstream effectors, including FLII. P-Rex1, via its scaffolding ability, binds to FLII through its GEL domain and brings it in close proximity to active Rac1 further stimulating the Rac1-FLII interaction via the LRR domain of FLII. Through this interaction, P-Rex1 induces phosphorylation of MLC (pMLC) thereby enhancing cell contraction in a FLII-dependent manner. This cascade accounts, in part, for P-Rex1-Rac1-driven cell migration. 
suggest that Tiam 1 activation of Racl is associated with reduced cell migration potentially through its ability to increase actin and cadherin deposition at cell-cell contacts, as previously suggested $^{14,30}$. The selective ability of Tiam1 to promote Rac1-dependent anti-migratory functions may be through regulation of the Racl interactome. Indeed, our proteomic analysis indicates an increased binding of Racl and IQGAP1 upon Tiam1 expression. Interestingly, active Racl has been shown to reduce the ability of IQGAP1 to interact with $\beta$ catenin $^{62}$, thereby counteracting the negative regulatory role of IQGAP1 on cadherin-mediated cell-cell adhesions ${ }^{63}$. In addition, active Rac1 suppresses IQGAP1-induced translocation of $\alpha$ catenin which precedes cell scattering in MDCKII cells ${ }^{64}$. The increased Rac1-IQGAP1 binding upon Tiam1 expression might therefore explain the anti-migratory phenotypic changes associated with these cells.

In contrast to Tiam1, P-Rex 1 expression in NIH3T3, A431 and MDCKII cells induces the formation of actin-rich membrane protrusions and cells exhibit reduced cell-cell contacts with an increased rate of cell migration and scattering. Consistent with our observations, P-Rex1-Rac1 signalling has been shown to drive cell migration in an immortalized primary human fibroblast cell line $\mathrm{e}^{31}$. In vivo studies also show that mice deficient in P-Rex1 have a decreased rate of melanoblast migration ${ }^{32}$. In addition, upregulation of P-Rexl has been implicated in promoting metastasis in prostate cancer as well as in melanoma ${ }^{32,33}$. Importantly, our data describes a novel signalling cascade involving P-Rex1, Racl and FLII that promotes cell migration. Evidence from the literature supports a crosstalk between FLII and small GTPases, where FLII has been shown to co-localize with Ras and other small GTPases at the leading edge of motile cells in actin-rich structures, including membrane ruffles and lamellipodia $^{46}$. FLII was shown to interact with Ras in vitro through the LRR domain ${ }^{65}$. We show that similarly to Ras, Rac1 binds to the LRR domain of FLII. In contrast, P-Rex1 binds to the GEL domain of FLII. These observations suggest that both P-Rexl and Racl could bind to FLII simultaneously forming a ternary complex that mediates P-Rex1-Rac1-driven cellular functions (Fig. 8e).

Intriguingly, FLII is considered a negative regulator of wound healing and cell migration ${ }^{47,53-56,66}$. However, we show that FLII is required for P-Rex1-Rac1-driven cell migration through modulating P-Rex1-mediated cell contraction. This indicates that, similarly to Rac1, FLII plays a dual role in regulating cell migration that is potentially dependent on the cellular context and FLII binding partners. Consistent with our data, fibroblasts from FLII heterozygous $\left(\mathrm{FLII}^{+/-}\right)$mice exhibit reduced contractility in collagen contraction assays ${ }^{47}$. Activation of myosin II through the phosphorylation of MLC has been directly implicated in cell migration through its role in promoting stress fibre formation and focal adhesion assembly in the centre of migrating cells. However, this process is mainly mediated through RhoA and its downstream effector ROCK ${ }^{57}$. Given that expression of P-Rex1 WT does not affect levels of active RhoA in migrating cells, together with the inability of ROCK inhibition to impede P-Rex1-driven cell migration, our findings support a RhoA-ROCK-independent pathway by which MLC phosphorylation and cell contraction can be modulated in cells, potentially at the leading edge where we observed co-localization of P-Rex1 and FLII. This is particularly interesting since Racl has been shown to regulate chemotaxis through its effector PAK1, which modulates the phosphorylation and localization of myosin IIB at the leading edge ${ }^{58}$. More recently, Racl activation has also been shown to stimulate MLC phosphorylation through inhibiting the MLC phosphatase ${ }^{59}$. Our data also indicates that P-Rexl-mediated cell contraction is important for collagen crosslinking and remodelling. We propose that P-Rex1-induced collagen remodelling is also dependent on FLII since FLII has recently been shown to interact with nonmuscle myosin IIA (NMMIIA) at cell protrusions, which is important for cell extension formation that enables FLII-dependent collagen remodelling by fibroblasts ${ }^{67}$. Given the link between ECM remodelling and cancer metastasis (reviewed in ref. 68), the role of P-Rex1 in collagen crosslinking and remodelling described in this manuscript may have important implications for P-Rex1-Racl-driven cancer cell invasion and metastasis.

In conclusion, our data provides clear evidence supporting a role of Tiam1 and P-Rex1 in driving differential signalling cascades downstream of Racl through modulating the Racl interactome. Characterization of the P-Rex1-FLII and Rac1-FLII interactions also highlights a novel role of P-Rex1 as a scaffolding protein to stimulate Rac1 pro-migratory phenotypes. Taken together our data indicate that P-Rex1 not only activates Rac1 but is also able to direct proteins, such as FLII, to the active form of Rac1 to mediate specific cellular functions, including cell contraction, that enable P-Rex1 to enhance cell migration. Therefore, this study provides insight into previously unreported P-Rex1-Rac1 cellular functions that mediate cell migration and highlights additional modes by which P-Rex1 and Rac1 might drive cancer dissemination.

\section{Methods}

Antibodies. Details of primary and secondary antibodies used in this study are summarized in Supplementary Table 2.

Cell culture. All cell lines were cultured at $37^{\circ} \mathrm{C}$ and $5 \% \mathrm{CO}_{2}$ using DMEM (Life Technologies, 61965-026) supplemented with 10\% v/v tetracycline-free fetal bovine serum (Tet-free FBS; Labtech International Ltd, FB-1001T/500) and $10 \mu \mathrm{g} \mathrm{ml}^{-1}$ penicillin-streptomycin (Life Technologies, 15140-122). Low glucose DMEM (1 $\mathrm{gl}^{-1}$; Life Technologies, 10567-014) was used for MDCKII cells. All experiments were conducted in full serum media supplemented with penicillinstreptomycin unless otherwise indicated. NIH3T3, A431, MDCKII, HEK293T, MCF7, CHL1 were obtained from ECACC, operated by Public Health England or ATCC. Primary human fibroblasts, described by Timpson et al. ${ }^{69}$ were used for this study.

Constructs. Details of plasmids used in this study are summarized in Supplementary Table 3.

Generation of cell lines. Transfection with the indicated constructs was performed using the TransIT-LT1 reagent (Mirus, MIR 2305) according to manufacturer's instructions. Retroviral transduction was performed by transfection of Phoenix packaging cells with retroviral constructs. Polybrene $\left(10 \mu \mathrm{g} \mathrm{ml}^{-1}\right.$; Sigma-Aldrich, 107689) was added to the cleared supernatant collected $24 \mathrm{~h}$ post transfection and the mixture was used to transduce cell lines. This was repeated three times with $24 \mathrm{~h}$ intervals using fresh supernatant each time. For antibiotic selection, cells transduced with the pRetroX-Tet-On Advanced plasmid only (control cells) were grown in tetracycline-free DMEM media with $1 \mathrm{mg} \mathrm{ml}^{-1} \mathrm{G} 418$ selection (Sigma-Aldrich, A1720). Cells with both the pRetroX-Tet-On Advanced and pRetroX-Tight-Pur (EV or with indicated proteins) were selected using $1 \mathrm{mg} \mathrm{ml}^{-1} \mathrm{G} 418$ and $2 \mu \mathrm{g} \mathrm{ml}^{-1}$ Puromycin (Sigma-Aldrich, P8833). NIH3T3 cells expressing histone-2B-GFP were subjected to $5 \mu \mathrm{g} \mathrm{ml}^{-1}$ blasticidin antibiotic selection (Sigma-Aldrich, 15205). Antibiotic selection was withdrawn during experiments. For induction of protein expression cells were treated with $1 \mu \mathrm{g} \mathrm{ml}^{-1}$ doxycycline $(+$ dox $)$ for $24-48 \mathrm{~h}$ before analysis. As a dox treatment control, cells were treated with an equal volume of $100 \%$ ethanol $(-$ dox $)$.

Transient transfection of siRNA. For knockdown of mouse FLII in NIH3T3 cells before ORIS migration assay or single-cell tracking, two FLII siRNA oligonucleotides ( $20 \mu \mathrm{M}$ stock; Sigma-Aldrich), outlined below, were prepared using the DharmaFECT 1 transfection reagent (GE Healthcare, T-2001-01) or the Lipofectamine RNAiMAX (Life Technologies, 137781) according to manufacturer's instructions and cells were reverse transfected. Cells were re-transfected $48 \mathrm{~h}$ later in the presence of ethanol $(-\mathrm{dox})$ or $1 \mu \mathrm{g} \mathrm{ml}{ }^{-1}$ dox $(+$ dox $)$ for an additional $24 \mathrm{~h}$. To determine the efficiency of siRNA transfection, levels of FLII were determined by western blot analysis. 
FLII siRNA 1 (Mm01_00187916) 5'-CAGAUCAACUACAAGCUCU[dT] $[\mathrm{dT}]-3^{\prime}$

FLII siRNA 2 (Mm01_00187920) 5'-GACUUUGAUGGGCUGCCUU[dT]

[dT]-3'

Transient silencing of human FLII in primary $\left(1^{\circ}\right)$ human fibroblasts and CHL1 cells and of human P-Rex1 in CHL1 and MCF7 cells was achieved by reverse transfection of the siRNA oligonucleotides, detailed below, from either Eurofins MWG operon or Qiagen using Lipofectamine RNAiMAX according to manufacturer's instructions. Western blot analysis was used to detect levels of FLII or P-Rex $148 \mathrm{~h}$ post transfection.

hFLII siRNA1 $5^{\prime}$-GCUGGAACACUUGUCUGUG[dT][dT]-3'

hFLII siRNA2 $5^{\prime}$ CAACCUGACCACGCUUCAU[dT][dT]- $3^{\prime}$

hP-siRNA5 Hs_P-Rex1_5 FlexiTube siRNA (Qiagen, 1027418-SI03144449)

$5^{\prime}$-CGAGUGUAACAGCAAUCGA[dT][dT]-3'

hP-siRNA6 Hs_P-Rex1_6 FlexiTube siRNA (Qiagen, 1027418-SI03246383)

$5^{\prime}$-GGGUCAGCCCACCCUUCAA[dT][dT]-3'

Transfection reagent alone (mock) or together with Dharmacon NT siRNA \#1 (GE Healthcare, D-001210-01-20) or siRNA\#4 (GE Healthcare, D-001210-04-20), were used as controls.

Analysis of cell morphology. Cells were seeded in the presence of ethanol ( - dox) or $1 \mu \mathrm{g} \mathrm{ml}^{-1}$ dox $(+$ dox $)$ for $24 \mathrm{~h}$ and phase-contrast images were taken. Cells were classified into three groups based on their morphology as outlined in Supplementary Methods.

Cell scattering assays. Cells were seeded at a concentration of $2 \times 10^{3}$ cells per well in a six-well plate for $24 \mathrm{~h}$ in DMEM media. Cells were then washed twice with phosphate-buffered saline (PBS; $137 \mathrm{mM} \mathrm{NaCl}, 2.7 \mathrm{mM} \mathrm{KCl}, 10 \mathrm{mM} \mathrm{Na}_{2} \mathrm{PO}_{4}$, $2 \mathrm{mM} \mathrm{KH}_{2} \mathrm{PO}_{4}$ in $\mathrm{dH}_{2} \mathrm{O}$ ) to remove any traces of serum followed by culturing in serum-free media (DMEM $+0.01 \%$ FBS) for $18-24 \mathrm{~h}$ before the addition of HGF $\left(10 \mathrm{ng} \mathrm{ml}^{-1}\right)$ or EGF $\left(100 \mathrm{ng} \mathrm{ml}^{-1}\right)$. Phase-contrast images of individual colonies were captured at 0,3 and $24 \mathrm{~h}$ post growth factor addition and used for quantification as outlined in Supplementary Methods.

ORIS migration assay. Cells were plated at a concentration of $5 \times 10^{5}$ cells per well in a six-well plate in the presence of ethanol $(-$ dox $)$ or $1 \mu \mathrm{g} \mathrm{ml}^{-1}$ dox $(+$ dox $) 24 \mathrm{~h}$ before assay. Cells were trypsinised, counted, and $\approx 5 \times 10^{5}$ cells were then incubated with Dic16 dye $(2.5 \mu \mathrm{M})$ for $30 \mathrm{~min}$ at $37^{\circ} \mathrm{C}$ and $5 \% \mathrm{CO}_{2}$. Dic16-labelled cells $\left(5 \times 10^{4}\right.$ cells per well) were seeded on fibronectin $\left(5 \mu \mathrm{g} \mathrm{ml}^{-1}\right.$; Sigma-Aldrich, F1141) coated 96-well plates fitted with stoppers (Platypus Technologies, CMA1.101) together with ethanol ( - dox $)$ or $1 \mu \mathrm{g} \mathrm{ml}^{-1}$ dox $(+$ dox $)$. Cells were incubated overnight in a humidity chamber at $37^{\circ} \mathrm{C}$ and $5 \% \mathrm{CO}_{2}$ before the removal of the stoppers. For analysis of cell migration following inhibition of cell contraction, cells were incubated with DMEM $+10 \%$ Tet-free FBS alone (control), or supplemented with the inactive enantiomer of blebbistatin [ $(+)$ blebbistatin] $(100 \mu \mathrm{M}$; Merk Millipore, 203392), blebbistatin [( \pm )-blebbistatin] (100 $\mu \mathrm{M}$; Merk Millipore, 203390) or the ROCK inhibitor Y27632 (10 $\mu \mathrm{M}$; SigmaAldrich, Y0503) for $24 \mathrm{~h}$ post stopper removal. Fluorescence images were taken using the low light microscope system $(\times 4$ and $\times 5$ magnification) at 0 and $24 \mathrm{~h}$ upon removal of stoppers and cell migration was quantified as outlined in Supplementary Methods.

Single-cell tracking. NIH3T3 cells expressing histone-2B-GFP were generated through reverse transfection with pBOS-Histone-2B-GFP. Cells were plated at a density of $5 \times 10^{4}$ cells per well into optically clear, flat-bottomed 96-well plates to achieve confluency. Confluent monolayers were wounded with a sterile $10 \mu \mathrm{l}$ pipette tip and 10\% Tet-free FBS supplemented DMEM was replaced, with thorough PBS washes before and after wounding. Images were captured and analysed as outlined in Supplementary Methods.

Fibroblast-collagen matrix contraction assay. $1^{\circ}$ human fibroblasts were transiently co-transduced with the respective pRetroX EV/GEF together with pRetroXTet-On Advanced to generate a transient dox-inducible system for expression of $\mathrm{EV}$ or the indicated GEF constructs. Fibroblast-collagen matrices were prepared as previously described by Timpson et al. ${ }^{69}$ with minor modifications. In brief, virally transduced cells were mixed with collagen $\mathrm{I}\left(2 \mathrm{mg} \mathrm{ml}^{-1}\right)$ in $3 \mathrm{ml} 10 \times \mathrm{MEM}$ and $\approx 3 \mathrm{ml} 0.22 \mathrm{M} \mathrm{NaOH}$ to achieve an alkaline mixture. The fibroblast-collagen mix (500 $\mu \mathrm{l}$ per well) was added gently to 24 -well plates and left to set at $37^{\circ} \mathrm{C}, 5 \% \mathrm{CO}_{2}$ before adding media containing ethanol ( - dox) or $1 \mu \mathrm{g} \mathrm{ml}^{-1}$ dox $(+$ dox) for $24 \mathrm{~h}$ to induce protein expression. Quantification of cell contraction was performed as outlined in Supplementary Methods.

Biochemical analysis of cell contraction. Transiently transduced $1^{\circ}$ human fibroblasts were plated at sub-confluency and $24 \mathrm{~h}$ later were treated with ethanol ( - dox $)$ or $1 \mu \mathrm{g} \mathrm{ml}^{-1}$ dox $(+$ dox $)$ for $24 \mathrm{~h}$. Cells were lysed in IP lysis buffer [50 mM Tris-HCL pH 7.5, $150 \mathrm{mM} \mathrm{NaCl}, 1 \%$ Triton-X-100 (v/v), 10\% glycerol (v/v), $2 \mathrm{mM}$ EDTA, $25 \mathrm{mM} \mathrm{NaF}, 2 \mathrm{mM} \mathrm{NaH} \mathrm{PO}_{4}, 1 \%$ protease inhibitor cocktail (Sigma-Aldrich, P8340) (v/v), 1\% phosphatase inhibitor cocktails 1 and 2
(Sigma-Aldrich, P5726, P0044) (v/v) in $\mathrm{dH}_{2} \mathrm{O}$ ] and lysates were mixed with equal volume of $2 \times$ SDS-polyacrylamide gel electrophoresis (PAGE) sample buffer [50\% NuPAGE LDS sample buffer $4 \times$ (Life Technologies, NP0008) (v/v), $20 \%$ NuPAGE sample reducing agent $10 \times$ (Life Technologies, NP0004) (v/v) in $\mathrm{dH}_{2} \mathrm{O}$. Levels of phosphorylated MLC (pMLC) were detected by western blot analysis. For analysis of pMLC in FLII depleted cells, hFLII siRNA1 was added to cells $24 \mathrm{~h}$ post plating for an additional $24 \mathrm{~h}$. Protein expression was then induced by dox for $24 \mathrm{~h}$ after which cells were lysed and analysed by western blot analysis. Western blot analysis was conducted $48 \mathrm{~h}$ post transfection with $\mathrm{hP}$-siRNA5 and hP-siRNA6 to analyse effect of P-Rex1 depletion on pMLC.

Histological analysis. Samples were fixed in $4 \%$ paraformaldehyde overnight before paraffin embedding. Histological staining was performed on 4 and $20 \mu \mathrm{m}$ sections deparaffinised in xylene and rehydrated using graded ethanol washes. Hematoxylin and eosin staining and counterstaining were undertaken on a Leica autostainer containing Hematoxylin reagent (Thermo Scientific, ASHB1000737AQ) and Eosin Y solution with Phloxine (Sigma-Aldrich, HT110332). A picrosirius red stain kit (Polysciences, 24901-250) was used for picrosirius staining. Mouse anti-pMLC (Ser19) (1:200; Cell Signaling, 3675) was used for pMLC immunohistochemistry staining.

Second harmonic generation (SHG) imaging of fibrillar collagen I. Hematoxylin and eosin paraffin sections $(20 \mu \mathrm{m})$ of fibroblast-collagen matrices were used for SHG imaging of fibrillar collagen. Images were captured and analysed as outlined in Supplementary Methods.

Gray-level co-occurrence matrix (GLCM) analysis. Stromal collagen fibre organization and crosslinking in fibroblast-collagen matrices was assessed using GLCM analysis. This method allows the study of the texture of a sample and provides a readout

of the level of crosslinked collagen organization, as previously described ${ }^{60,61,70}$. GLCM analysis was performed in ImageJ software as outlined in Supplementary Methods.

Immunofluorescence. Cells grown on coverslips in the presence of ethanol ( - dox) or $1 \mu \mathrm{g} \mathrm{ml}^{-1}$ dox $(+$ dox $)$ for $24 \mathrm{~h}$ were fixed in $4 \%$ formaldehyde for $15 \mathrm{~min}$ and incubated for $3 \mathrm{~min}$ with cell permeabilization buffer $(0.5 \%$ Triton-X $(\mathrm{v} / \mathrm{v})$ in PBS) at room temperature. Cells were next blocked in $1 \%$ bovine serum albumin (BSA; Roche, 10735078001) in PBS for 30 min before incubation with relevant primary antibodies (in $1 \%$ BSA) for 1 hour at room temperature. The respective fluorophore-conjugated secondary antibodies were then added for an additional hour at room temperature in the dark. Antibodies used for immunofluorescence are outlined in Supplementary Table 2. Coverslips were mounted onto slides using the ProLong Gold antifade reagent with DAPI stain (Invitogen, P36935) and images were captured using the low light microscope system and processed with Metamorph software (Molecular Devices). Images shown were analysed in ImageJ. Additional details on the specifications of microscopes used for image recording are described in Supplementary Methods.

Duolink in situ proximity ligation assay. Cells seeded on glass coverslips in the presence of $1 \mu \mathrm{g} \mathrm{ml}^{-1}$ dox for $24 \mathrm{~h}$ were fixed in $4 \%$ formaldehyde and subjected to Duolink in situ PLA using mouse anti-Rac1 antibody (1:100; BD Biosciences, 610650) and rabbit anti-FLII antibody (1:100; Sigma-Aldrich, HPA007084), the respective Duolink in situ PLA probes (Olink Bioscience, anti-mouse 92004-0100, anti-rabbit 92002-0100) and the Duolink in situ detection reagent kit (Olink Bioscience, 92013-0100, 92014-0100) according to manufacturer's instructions. For CHL1 cells, mouse anti-FLII antibody (1:100; Santa Cruz, sc-21716) and rabbit anti-P-Rex1 antibody (1:100; Sigma-Aldrich, HPA001927) were used for the Duolink in situ PLA analysis. Coverslips were mounted onto slides using ProLong Gold antifade reagent with DAPI stain (Life Technologies, P36935) and images were taken using the low light microscope system ( $\times 100$ magnification). Phalloidin and DAPI were used as fluorescence markers against the actin cytoskeleton and the nucleus, respectively. Quantification of the Duolink signal was performed as outlined in Supplementary Methods.

Active GTPase pulldown. NIH3T3 cells lysed in GST lysis buffer [ $25 \mathrm{mM}$ Tris-HCL pH 7.2, $150 \mathrm{mM} \mathrm{NaCl}, 5 \mathrm{mM} \mathrm{MgCl} 2,1 \%$ Nonidet P40 (v/v), 5\% glycerol $(\mathrm{v} / \mathrm{v}), 1 \%$ protease inhibitor cocktail $(\mathrm{v} / \mathrm{v}), 1 \%$ phosphatase inhibitor cocktails 1 and $2(\mathrm{v} / \mathrm{v})$ in $\mathrm{dH}_{2} \mathrm{O}$ ] were divided equally and subjected to either biotinylated PAK-CRIB pulldown or GST-Rhotekin pulldown to detect levels of active Rac1/Cdc42 and RhoA, respectively. For measuring Rac1 and Cdc42 activity, lysates were incubated with $60 \mu \mathrm{l}$ Strep-Tactin superflow resin (IBA GmbH, 21206-10) together with $6 \mu \mathrm{g}$ biotinylated PAK-CRIB purified peptide for 1 hour at $4{ }^{\circ} \mathrm{C}$. For measuring RhoA activity the active RhoA pulldown and detection kit (Thermo Scientific, 16116) was used following manufacturer's instructions. GDP and GTP $\gamma$ loaded NIH3T3 lysates were used as negative and positive active GTPase pulldown controls, respectively. Levels of active and total Rac1, Cdc42 and RhoA 
were detected by western blot analysis using the respective primary antibodies. To detect levels of active RhoA in migrating cells, P-Rex1 WT expressing NIH3T3 cells were plated in confluent monolayers that were subjected to multiple scratches to stimulate migration. Lysates were prepared $24 \mathrm{~h}$ post scratching and dox induction and analysed using the active RhoA pulldown and detection kit.

Active RhoG ELISA. NIH3T3 cells were plated in confluent monolayers that were subjected to multiple scratches to stimulate migration. Lysates were prepared $24 \mathrm{~h}$ post scratching and dox induction and analysed using the RhoG ELISA kit (Biosource, MBS9324983) according to manufacturer's instructions.

GST-pulldown. GST or GST-Rac1 WT immobilized on Glutathione Sepharose 4B (GE Healthcare, 17-0756-01) were washed using GST lysis buffer followed by nucleotide chelating using $0.5 \mathrm{M}$ EDTA $(10 \mathrm{mM})$ and incubation with Guanosine $5^{\prime}$-diphosphate sodium salt (GDP; $100 \mu \mathrm{M}$; Sigma-Aldrich, G7127) or Guanosine $5^{\prime}$-O-(3-thiotriphosphate) tetralithium salt (GTP $\gamma \mathrm{S} ; 1 \mathrm{mM}$; Sigma-Aldrich, G8634) for $15 \mathrm{~min}$ at $30^{\circ} \mathrm{C}$. Termination of the reaction was achieved by adding $1 \mathrm{M}$ $\mathrm{MgCl}_{2}(60 \mathrm{mM})$. HEK293T cells were lysed in GST lysis buffer and equal protein amounts were incubated with GDP or GTP $\gamma$ S loaded GST and GST-Rac1 WT beads for $2 \mathrm{~h}$ at $4{ }^{\circ} \mathrm{C}$. Beads were washed following incubation and eluted using $20 \mu \mathrm{l} 2 \times$ SDS-PAGE sample buffer for western blot analysis.

In vitro protein interaction. GST or GST-FLII GEL immobilized on Glutathione Sepharose $4 \mathrm{~B}$ were washed using GST lysis buffer before incubation with $1 \mu \mathrm{g}$ recombinant P-Rexl full-length protein for $2 \mathrm{~h}$ at $4{ }^{\circ} \mathrm{C}$. Beads were washed following incubation and eluted using $20 \mu \mathrm{l} 2 \mathrm{x}$ SDS-PAGE sample buffer for western blot analysis.

Immunoprecipitation and pulldowns. Cells were lysed in GST lysis buffer or SF-TAP lysis buffer [ $30 \mathrm{mM}$ Tris pH 7.4, $150 \mathrm{mM} \mathrm{NaCl}, 0.5 \%$ Nonidet $\mathrm{P} 40$ (v/v), $1 \%$ protease inhibitor cocktail $(\mathrm{v} / \mathrm{v}), 1 \%$ phosphatase inhibitor cocktails 1 and 2 $(\mathrm{v} / \mathrm{v})$ in $\mathrm{dH}_{2} \mathrm{O}$. For immunoprecipitation, lysates were incubated with $20-50 \mu \mathrm{l}$ antibody pre-bound to GammaBind G Sepharose beads (GE Healthcare, 17-088501 ) for $1-2 \mathrm{~h}$ at $4{ }^{\circ} \mathrm{C}$, washed and resuspended in $20-25 \mu \mathrm{l}$ of $2 \mathrm{x}$ SDS-PAGE sample buffer for western blot analysis. For Strep pulldown, lysates were incubated with $200 \mu \mathrm{l} \mathrm{Strep-Tactin} \mathrm{superflow} \mathrm{resin} \mathrm{for} 90 \mathrm{~min}$ at $4^{\circ} \mathrm{C}$, washed and then eluted by incubating with $500 \mu \mathrm{l}$ desthiobiotin elution buffer $(2.5 \mathrm{mM}$; Sigma-Aldrich, D1411) for $30 \mathrm{~min}$ at $4{ }^{\circ} \mathrm{C}$. For FLAG immunoprecipitation, lysates were incubated with $50 \mu \mathrm{l}$ anti-FLAG M2 affinity gel (Sigma-Aldrich, A2220) for $75 \mathrm{~min}$ at $4{ }^{\circ} \mathrm{C}$, washed and then eluted using the 3xFLAG peptide $\left(200 \mathrm{\mu g} \mathrm{ml}^{-1}\right.$; Sigma-Aldrich, F4799). Amicon filter units with a $3 \mathrm{kDa}$ protein cutoff (EMD Millipore, UFC500396) were used to concentrate the Strep-Tactin superflow and FLAG eluates. Concentrated protein samples were mixed with $20 \mu \mathrm{l} 2 \times$ SDS-PAGE sample buffer for western blot analysis. Details of antibodies and commercial conjugated beads used for immunoprecipitation and pulldowns in this study are listed in Supplementary Table 2.

\section{StrepII-FLAG tandem affinity purification. The StrepII-FLAG (SF-TAP) was} performed as outlined by Gloeckner et al. ${ }^{36}$ with minor modifications. In brief, cells transduced with the dox-inducible system for expressing SF-Racl alone or together with the indicated GEFs were treated with either ethanol ( - dox $)$ or $1 \mu \mathrm{g} \mathrm{ml}^{-1}$ dox $(+$ dox $)$ and incubated for $48 \mathrm{~h}$. Cells were then lysed in SF-TAP lysis buffer and equal protein amounts were subjected to a Strep pulldown as detailed above. Eluates were then subjected to FLAG immunoprecipitation as outlined earlier. Amicon filter units with a $3 \mathrm{kDa}$ protein cutoff were used to concentrate the FLAG eluates. Concentrated protein samples were mixed with $20 \mu \mathrm{l} 2 \times$ SDS-PAGE sample buffer and analysed by western blot analysis or stored at $-80^{\circ} \mathrm{C}$ for later use.

\begin{abstract}
Western blot analysis. Samples were prepared as indicated in the respective experimental sections and were resolved using NuPAGE Novex Tris-Acetate pre-cast gels (Life Technologies, 3-8\% 10-well EA0375BOX, 3-8\% 12-well EA03752BOX) or NuPAGE Novex Bis-Tris pre-cast gels (Life Technologies, 12\% 10-well NP0341BOX, 12\% 12-well NP0342BOX, 4-12\% 10-well NP0321BOX, 12-well NP0321BOX). The BLUeye Pre-Stained Protein Ladder (Geneflow Ltd, S6-0024) was run alongside the samples for protein size reference. Proteins were transferred from gels onto Immobolin PVDF membranes (Millipore, IPFL00010). Western blot analysis was performed using antibodies listed in Supplementary Table 2 as outlined therein and visualized on Hyperfilm ECL (GE Healthcare, 28-9068) using the ECL western blotting analysis system (GE Healthcare, RPN2109). Adobe Photoshop software was used to crop full blots (Supplementary Figs 8 and 9). Band intensities were quantified using the ImageJ Gel analysis tool.
\end{abstract}

SILAC labelling and protein identification. Stable isotope labelling by amino acids in cell culture (SILAC) media was prepared using high glucose DMEM without lysine $(\mathrm{K})$ or arginine (R) (PAA) supplemented with $10 \%$ dialysed FBS (Life Technologies, 26400-044), 1\% L-glutamine (Sigma-Aldrich, G7513) and
$10 \mu \mathrm{g} \mathrm{ml}^{-1}$ penicillin-streptomycin. For labelling, cells were cultured for six doubling rounds using three different SILAC culturing media prepared using different amino acids stock solutions as outlined in Supplementary Tables 4 and 5. The efficiency of SILAC labelling was evaluated by determining the amino acid incorporation rate as outlined in Supplementary Methods. For protein identification by mass spectrometry, concentrated mixed SF-TAP eluates were prepared and processed as detailed in Supplementary Methods

Ingenuity analysis and heat map generation. Information obtained from the SILAC screens were used to generate a heat map representing a cluster of Racl binding partners falling under one of the following IPA functional groups: cell movement, cell morphology, cell signalling, cell-to-cell signalling and interaction, as well as cell assembly and organization. The heat map, generated using the $\mathrm{R}$ Project software, represents the $\log 2$ of SILAC protein ratios relative to control cells expressing SF-Rac1 alone from a representative SILAC and reverse SILAC experiment.

Statistical analysis. For assessing statistical significance between two sets of data a two-tailed paired or two-sample equal variance student's $t$-test was conducted with $P$ value $\leq 0.05$ considered significant and $P$ value $\leq 0.01$ considered highly significant. Test and $P$ values are specified in figure legends where appropriate.

\section{References}

1. Geiger, T. R. \& Peeper, D. S. Metastasis mechanisms. Biochim. Biophys. Acta 1796, 293-308 (2009).

2. Yilmaz, M. \& Christofori, G. Mechanisms of motility in metastasizing cells. Mol. Cancer Res. 8, 629-642 (2010).

3. Parri, M. \& Chiarugi, P. Rac and Rho GTPases in cancer cell motility control. Cell Commun. Signal. 8, 23 (2010).

4. Lauffenburger, D. A. \& Horwitz, A. F. Cell migration: a physically integrated molecular process. Cell 84, 359-369 (1996).

5. Malliri, A. \& Collard, J. G. Role of Rho-family proteins in cell adhesion and cancer. Curr. Opin. Cell Biol. 15, 583-589 (2003).

6. Mack, N. A., Whalley, H. J., Castillo-Lluva, S. \& Malliri, A. The diverse roles of Rac signaling in tumorigenesis. Cell Cycle 10, 1571-1581 (2011).

7. Williamson, R. C. et al. Coronin-1C and RCC2 guide mesenchymal migration by trafficking Rac1 and controlling GEF exposure. J. Cell Sci. 127, 4292-4307 (2014).

8. Braun, A. et al. Rac1 and Aurora A regulate MCAK to polarize microtubule growth in migrating endothelial cells. J. Cell Biol. 206, 97-112 (2014).

9. Miki, H., Yamaguchi, H., Suetsugu, S. \& Takenawa, T. IRSp53 is an essential intermediate between Rac and WAVE in the regulation of membrane ruffling. Nature 408, 732-735 (2000).

10. Takenawa, T. \& Miki, H. WASP and WAVE family proteins: key molecules for rapid rearrangement of cortical actin filaments and cell movement. J. Cell Sci. 114, 1801-1809 (2001).

11. Johnston, S. A., Bramble, J. P., Yeung, C. L., Mendes, P. M. \& Machesky, L. M. Arp2/3 complex activity in filopodia of spreading cells. BMC. Cell. Biol. 9, 65 (2008).

12. Price, L. S., Leng, J., Schwartz, M. A. \& Bokoch, G. M. Activation of Rac and Cdc42 by integrins mediates cell spreading. Mol. Biol. Cell $\mathbf{9}$, 1863-1871 (1998).

13. Rottner, K., Hall, A. \& Small, J. V. Interplay between Rac and Rho in the control of substrate contact dynamics. Curr. Biol. 9, 640-648 (1999).

14. Hordijk, P. L. et al. Inhibition of invasion of epithelial cells by Tiam1-Rac signaling. Science 278, 1464-1466 (1997).

15. Nola, S. et al. Ajuba is required for Rac activation and maintenance of E-cadherin adhesion. J. Cell Biol. 195, 855-871 (2011).

16. Dawson, J. C., Bruche, S., Spence, H. J., Braga, V. M. \& Machesky, L. M. Mtss1 promotes cell-cell junction assembly and stability through the small GTPase Rac1. PLoS ONE 7, e31141 (2012).

17. Jin, G. et al. Biomechanical regulation of matrix metalloproteinase-9 in cultured chondrocytes. J. Orthop. Res. 18, 899-908 (2000).

18. Westermarck, J. \& Kahari, V. M. Regulation of matrix metalloproteinase expression in tumor invasion. FASEB J. 13, 781-792 (1999).

19. Lozano, E., Betson, M. \& Braga, V. M. Tumor progression: small GTPases and loss of cell-cell adhesion. Bioessays 25, 452-463 (2003).

20. Etienne-Manneville, S. \& Hall, A. Rho GTPases in cell biology. Nature 420, 629-635 (2002).

21. Sander, E. E. \& Collard, J. G. Rho-like GTPases: their role in epithelial cell-cell adhesion and invasion. Eur. J. Cancer. 35, 1905-1911 (1999).

22. Zhou, K. et al. Guanine nucleotide exchange factors regulate specificity of downstream signaling from Rac and Cdc42. J. Biol. Chem. 273, 16782-16786 (1998).

23. Krause, S. A. et al. Functional specialisation of yeast Rho1 GTP exchange factors. J. Cell. Sci. 125, 2721-2731 (2012). 
24. Connolly, B. A., Rice, J., Feig, L. A. \& Buchsbaum, R. J. Tiam1-IRSp53 complex formation directs specificity of rac-mediated actin cytoskeleton regulation. Mol. Cell. Biol. 25, 4602-4614 (2005).

25. Rajagopal, S. et al. Scaffold proteins IRSp53 and spinophilin regulate localized Rac activation by T-lymphocyte invasion and metastasis protein 1 (TIAM1). J. Biol. Chem. 285, 18060-18071 (2010).

26. Manser, E. et al. PAK kinases are directly coupled to the PIX family of nucleotide exchange factors. Mol. Cell. 1, 183-192 (1998).

27. Buchsbaum, R. J., Connolly, B. A. \& Feig, L. A. Interaction of Rac exchange factors Tiam1 and Ras-GRF1 with a scaffold for the p38 mitogen-activated protein kinase cascade. Mol. Cell. Biol. 22, 4073-4085 (2002).

28. Buchsbaum, R. J., Connolly, B. A. \& Feig, L. A. Regulation of p70 S6 kinase by complex formation between the Rac guanine nucleotide exchange factor (Rac-GEF) Tiam1 and the scaffold spinophilin. J. Biol. Chem. 278, 18833-18841 (2003).

29. Sander, E. E., ten Klooster, J. P., van Delft, S., van der Kammen, R. A. \& Collard, J. G. Rac downregulates Rho activity: reciprocal balance between both GTPases determines cellular morphology and migratory behavior. J. Cell Biol. 147, 1009-1022 (1999).

30. Uhlenbrock, K. et al. The RacGEF Tiam1 inhibits migration and invasion of metastatic melanoma via a novel adhesive mechanism. J. Cell. Sci. 117, 4863-4871 (2004).

31. Campbell, A. D. et al. P-Rex1 cooperates with PDGFRbeta to drive cellular migration in 3D microenvironments. PLoS ONE 8, e53982 (2013).

32. Lindsay, C. R. et al. P-Rex1 is required for efficient melanoblast migration and melanoma metastasis. Nat. Commun. 2, 555 (2011).

33. Qin, J. et al. Upregulation of PIP3-dependent Rac exchanger 1 (P-Rex1) promotes prostate cancer metastasis. Oncogene 28, 1853-1863 (2009).

34. Nie, B., Cheng, N., Dinauer, M. C. \& Ye, R. D. Characterization of P-Rex1 for its role in fMet-Leu-Phe-induced superoxide production in reconstituted COS(phox) cells. Cell. Signal. 22, 770-782 (2010).

35. Tolias, K. F. et al. The Rac1-GEF Tiam1 couples the NMDA receptor to the activity-dependent development of dendritic arbors and spines. Neuron 45 , 525-538 (2005)

36. Gloeckner, C. J., Boldt, K., Schumacher, A., Roepman, R. \& Ueffing, M. A novel tandem affinity purification strategy for the efficient isolation and characterisation of native protein complexes. Proteomics 7, 4228-4234 (2007).

37. Gloeckner, C. J., Boldt, K., Schumacher, A. \& Ueffing, M. Tandem affinity purification of protein complexes from mammalian cells by the Strep/FLAG (SF)-TAP tag. Methods Mol. Biol. 564, 359-372 (2009).

38. Gloeckner, C. J., Boldt, K. \& Ueffing, M. Strep/FLAG tandem affinity purification (SF-TAP) to study protein interactions. Curr. Protoc. Protein Sci. 57, 19 20.1-19.20.19 (2009).

39. Ong, S. E. et al. Stable isotope labeling by amino acids in cell culture, SILAC, as a simple and accurate approach to expression proteomics. Mol. Cell. Proteomics 1, 376-386 (2002).

40. Ong, S. E. \& Mann, M. Mass spectrometry-based proteomics turns quantitative. Nat. Chem. Biol. 1, 252-262 (2005).

41. Prasad, T. S., Kandasamy, K. \& Pandey, A. Human protein reference database and human proteinpedia as discovery tools for systems biology. Methods Mol. Biol. 577, 67-79 (2009)

42. Kandasamy, K. et al. NetPath: a public resource of curated signal transduction pathways. Genome Biol. 11, R3 (2010).

43. McDowall, M. D., Scott, M. S. \& Barton, G. J. PIPs: human proteinprotein interaction prediction database. Nucleic Acids Res. 37, D651-D656 (2009).

44. Scott, M. S. \& Barton, G. J. Probabilistic prediction and ranking of human protein-protein interactions. BMC Bioinformatics 8, 239 (2007).

45. Mann, M. Functional and quantitative proteomics using SILAC. Nat. Rev. Mol. Cell Biol. 7, 952-958 (2006).

46. Davy, D. A., Campbell, H. D., Fountain, S., de Jong, D. \& Crouch, M. F. The flightless I protein colocalizes with actin- and microtubule-based structures in motile Swiss 3T3 fibroblasts: evidence for the involvement of PI 3-kinase and Ras-related small GTPases. J. Cell Sci. 114, 549-562 (2001).

47. Kopecki, Z., O’Neill, G. M., Arkell, R. M. \& Cowin, A. J. Regulation of focal adhesions by flightless $\mathrm{i}$ involves inhibition of paxillin phosphorylation via a Rac1-dependent pathway. J. Invest. Dermatol. 131, 1450-1459 (2011).

48. Silacci, P. et al. Gelsolin superfamily proteins: key regulators of cellular functions. Cell. Mol. Life Sci. 61, 2614-2623 (2004).

49. Kobe, B. \& Kajava, A. V. The leucine-rich repeat as a protein recognition motif. Curr. Opin. Struct. Biol. 11, 725-732 (2001).

50. Kobe, B. \& Deisenhofer, J. Proteins with leucine-rich repeats. Curr. Opin. Struct. Biol. 5, 409-416 (1995).

51. Liu, Y. T. \& Yin, H. L. Identification of the binding partners for flightless I, a novel protein bridging the leucine-rich repeat and the gelsolin superfamilies. $J$. Biol. Chem. 273, 7920-7927 (1998).
52. Lee, Y. H., Campbell, H. D. \& Stallcup, M. R. Developmentally essential protein flightless $\mathrm{I}$ is a nuclear receptor coactivator with actin binding activity. Mol. Cell. Biol. 24, 2103-2117 (2004).

53. Cowin, A. J. et al. Flightless I deficiency enhances wound repair by increasing cell migration and proliferation. J. Pathol. 211, 572-581 (2007).

54. Lin, C. H., Waters, J. M., Powell, B. C., Arkell, R. M. \& Cowin, A. J. Decreased expression of Flightless I, a gelsolin family member and developmental regulator, in early-gestation fetal wounds improves healing. Mamm. Genome 22, 341-352 (2011)

55. Kopecki, Z., Arkell, R., Powell, B. C. \& Cowin, A. J. Flightless I regulates hemidesmosome formation and integrin-mediated cellular adhesion and migration during wound repair. J. Invest. Dermatol. 129, 2031-2045 (2009).

56. Mohammad, I. et al. Flightless I is a focal adhesion-associated actincapping protein that regulates cell migration. FASEB J. 26, 3260-3272 (2012).

57. Totsukawa, G. et al. Distinct roles of ROCK (Rho-kinase) and MLCK in spatial regulation of MLC phosphorylation for assembly of stress fibers and focal adhesions in 3 T3 fibroblasts. J. Cell Biol. 150, 797-806 (2000).

58. Even-Faitelson, L., Rosenberg, M. \& Ravid, S. PAK1 regulates myosin II-B phosphorylation, filament assembly, localization and cell chemotaxis. Cell. Signal. 17, 1137-1148 (2005).

59. Shibata, K. et al. Rac1 regulates myosin II phosphorylation through regulation of myosin light chain phosphatase. J. Cell. Physiol. 230, 1352-1364 (2014).

60. Nobis, M. et al. Intravital FLIM-FRET imaging reveals dasatinibinduced spatial control of src in pancreatic cancer. Cancer Res. 73, 4674-4686 (2013).

61. Cicchi, R. et al. Scoring of collagen organization in healthy and diseased human dermis by multiphoton microscopy. J Biophotonics 3, 34-43 (2010).

62. Fukata, M. et al. Cdc42 and Racl regulate the interaction of IQGAP1 with betacatenin. J. Biol. Chem. 274, 26044-26050 (1999).

63. Kuroda, S. et al. Role of IQGAP1, a target of the small GTPases Cdc42 and $\mathrm{Rac1}$, in regulation of E-cadherin-mediated cell-cell adhesion. Science 281, 832-835 (1998)

64. Fukata, M. et al. Involvement of IQGAP1, an effector of Rac1 and Cdc42 GTPases, in cell-cell dissociation during cell scattering. Mol. Cell. Biol. 21, 2165-2183 (2001).

65. Goshima, M. et al. Characterization of a novel Ras-binding protein Ce-FLI-1 comprising leucine-rich repeats and gelsolin-like domains. Biochem. Biophys. Res. Commun. 257, 111-116 (1999).

66. Adams, D. H. et al. Gender specific effects on the actin-remodelling protein Flightless I and TGF-betal contribute to impaired wound healing in aged skin. Int. J. Biochem. Cell Biol. 40, 1555-1569 (2008).

67. Arora, P. D., Wang, Y., Bresnick, A., Janmey, P. A. \& McCulloch, C. A. Flightless I interacts with NMMIIA to promote cell extension formation, which enables collagen remodeling. Mol. Biol. Cell 26, 2279-2297 (2015).

68. Bonnans, C., Chou, J. \& Werb, Z. Remodelling the extracellular matrix in development and disease. Nat. Rev. Mol. Cell Biol. 15, 786-801 (2014).

69. Timpson, P. et al. Organotypic collagen I assay: a malleable platform to assess cell behaviour in a 3-dimensional context. J. Vis. Exp. 56, e3089 (2011).

70. Huo, C. W. et al. High mammographic density is associated with an increase in stromal collagen and immune cells within the mammary epithelium. Breast Cancer Res. 17, 79 (2015).

\section{Acknowledgements}

We thank Heidi Welch for kindly providing P-Rexl full-length recombinant protein, Christopher McCulloch for kindly providing the GST-FLII GEL expression vector, Michael R. Stallcup for kindly providing the FLAG-tagged FLII domain mutant constructs and Marius Ueffing for kindly providing the SF-TAP tag construct. We also thank Hui Sun Leong (RNA biology group, CRUKMI) for generating the heat map of Racl binding partners using the $\mathrm{R}$ project software and members of the Cell Signalling laboratory for critical reading of the manuscript. This work was supported by Cancer Research UK (grant number C5759/A12328) for A.M., the National Health and Medical Research (NHMRC), the Australian Research Council (ARC) and the Cancer Council NSW for P.T.

\section{Author contributions}

H.M. designed and performed the majority of the experiments and analysis and wrote the manuscript. A.C. performed the mass spectrometry analysis on samples submitted following SILAC labelling and SF-TAP. A.W. performed single-cell tracking experiments in P-Rex1 WT expressing cells following FLII depletion and analysed the localization of P-Rexl and FLII in CHL1 migrating cells as well as processed CHL1 lysates following FLII depletion by siRNA. C.V. analysed fibroblast-collagen matrices following contraction assays by performing pMLC immunohistochemistry, picrosirius staining, SHG imagining and GLCM modelling. G.W. performed the in vitro interaction between 
P-Rex1 and FLII GEL, examined the role of endogenous P-Rex1 in regulating pMLC levels in MCF7 and CHL1 cells and analysed levels of active RhoG upon P-Rex1 expression in migrating cells. P.T. provided the expertise and equipment required for analysing the fibroblast-collagen matrices following contraction assays. B.M. provided the expertise and equipment required for the mass spectrometry analysis. A.M. devised and supervised the project and edited the manuscript.

\section{Additional information}

Supplementary Information accompanies this paper at http://www.nature.com/ naturecommunications

Competing financial interests: The authors declare no competing financial interests.
Reprints and permission information is available online at http://npg.nature.com/ reprintsandpermissions/

How to cite this article: Marei, H. et al. Differential Rac1 signalling by guanine nucleotide exchange factors implicates FLII in regulating Rac1-driven cell migration. Nat. Commun. 7:10664 doi: 10.1038/ncomms10664 (2016).

(c) (i) This work is licensed under a Creative Commons Attribution 4.0 International License. The images or other third party material in this article are included in the article's Creative Commons license, unless indicated otherwise in the credit line; if the material is not included under the Creative Commons license, users will need to obtain permission from the license holder to reproduce the material. To view a copy of this license, visit http://creativecommons.org/licenses/by/4.0/ 